\title{
PTF1a Activity in Enriched Posterior Foregut Endoderm, but Not Definitive Endoderm, Leads to Enhanced Pancreatic Differentiation in an In Vitro Mouse ESC-Based Model
}

\author{
Gopika G. Nair ${ }^{1,2}$ and Jon S. Odorico ${ }^{1}$ \\ ${ }^{1}$ Division of Transplantation, Department of Surgery, University of Wisconsin-Madison School of Medicine and Public Health, \\ Madison, WI 53792, USA \\ ${ }^{2}$ Diabetes Research Center, University of California-San Francisco, San Francisco, CA 94143, USA \\ Correspondence should be addressed to Jon S. Odorico; jon@surgery.wisc.edu
}

Received 30 July 2015; Revised 7 November 2015; Accepted 26 November 2015

Academic Editor: Bruno Becker

Copyright (C) 2016 G. G. Nair and J. S. Odorico. This is an open access article distributed under the Creative Commons Attribution License, which permits unrestricted use, distribution, and reproduction in any medium, provided the original work is properly cited.

\begin{abstract}
Transcription factors are tools repetitively used by the embryo to generate a variety of lineages. Hence, their context of activation is an important determinant of their ability to specifically trigger certain cell fates, but not others. The context is also consequential when considering directing differentiation of embryonic stem cells (ESCs). In this study, we sought to assess the context of pancreatic transcription factor la (PTFla) activation in reference to its propancreatic effects in mouse ESCs (mESCs). We hypothesized that an enriched endodermal population would respond to PTFla and trigger the pancreatic program more effectively than a spontaneously differentiated population. Using an in vitro model of pancreas development that we recently established, we found that inducing PTFla in highly enriched definitive endoderm did not promote pancreatic differentiation but induction in more differentiated endoderm, specifically posterior foregut endoderm, did form pancreatic progenitors. These progenitors never underwent terminal differentiation to endocrine or acinar phenotype. However, a short 3D culture period, prior to PTFla induction, led to the generation of monohormonal insulin ${ }^{+}$cells and amylase-expressing cells. Our findings suggest that enriched posterior foregut endoderm is competent to respond to PTFla's propancreatic activity; but a 3D culture environment is essential for terminal differentiation of pancreatic progenitors.
\end{abstract}

\section{Introduction}

ESCs hold great potential in regenerative medicine due to their unlimited ability to self-renew and differentiate to a repertoire of lineages and hence have been the focus of many differentiation studies to obtain transplantable cell types. For example, making functional $\beta$ cells successfully promises a cure for Type 1 Diabetes. Definitive endoderm (DE) is the gastrulation-derived cell population that ultimately gives rise to the respiratory and digestive tract organs, including the pancreas. Therefore, efforts to generate functional $\beta$ cells involve directed differentiation of ESCs to DE followed by stepwise differentiation to pancreatic cells, inspired by processes from normal pancreatic development.

Several studies have used TGF- $\beta$ family molecules such as Activin A, Nodal, and BMP4 [1-6] or small molecules
$[7,8]$ that mimic endogenous nodal signaling to specify endodermal fate in mouse and human ESCs. Transcription factors that are activated by Nodal signaling include Mixlike homeodomain proteins, Gata zinc finger factors, Sox HMG domain proteins, and Fox forkhead factors [9]. Many genes expressed in $\mathrm{DE}$ are also expressed in mesoderm, neuroectoderm, and extraembryonic endoderm (EE). For example, Foxa 2 is expressed in both DE and mesoderm; Sox 17 is expressed in DE and EE, and hence there is no single marker to identify DE. Nonetheless, the DE population is marked by the coexpression of FOXA2 [10] and SOX17 [11] though individually both of these markers are not specific for DE. Due to heterogeneity in ESC differentiation cultures, the presence of DE markers and the absence of markers of nontarget cell types are used to determine DE-enriched populations. Further differentiation of DE to pancreatic cell 
types has been reported using a cocktail of growth factors, including FGF10, FGF7, and RA, and inhibitors of key signaling pathways, including Noggin, KAAD-cyclopamine, SANT-1, and Alk5 inhibitor [12-16].

However, current ESC to $\beta$ cell differentiation protocols are limited by low efficiency and generation of immature polyhormonal cells as well as a formation of not-so-robust glucose responsive cells $[12,17-21]$. This leads us to believe that some important transcriptional events that are necessary for proper pancreatic development are missing. PTFla, a critical determinant of pancreatic fate, is not rigorously expressed/is lacking in many of the published differentiation protocols $[12,13,18,20,22]$. The significance of the role of PTFla in committing foregut endodermal cells to pancreatic lineage was elucidated by acquisition of a duodenal fate by pancreatic progenitors that lacked PTFla in murine transgenic lineage tracing systems [23]. We have previously shown that ectopic expression of PTFla in mouse ESCs can be used to model pancreas development in vitro and results in the generation of monohormonal endocrine cells embedded in exocrine tissue [24]. However, the correct context of PTFla signaling that is sufficient to direct differentiation to the pancreatic lineage has not been investigated in ESCs until now, and it would be of interest to test if PTFla signaling can overcome the deficiencies in the current methods of differentiation. In this study, we addressed this question using our in vitro model of pancreas development, wherein PTFla was induced in populations of cells exclusively differentiated to DE or its derivatives.

\section{Materials and Methods}

2.1. Cell Lines. The generation and characterization of TetPtfla line that was used in this study are described in [24].

\section{2. mESC Maintenance and Differentiation. Tet-Ptfla ESCs} were maintained in an undifferentiated state on MEF feeder layers with LIF in DMEM-high glucose with 15\% FBS, $100 \mathrm{U}$ penicillin/streptomycin, $2 \mathrm{mM}$ L-glut, $2 \mathrm{mM}$ NEAA, $1 \mathrm{mM}$ Sodium pyruvate, and $0.05 \mathrm{mM} \beta$-mercaptoethanol and cultured in differentiation media as previously described [26], with the following modifications. Media compositions of various differentiation protocols that we tested are enumerated in Table 1 . Initial cell seeding density is also indicated for every protocol. The growth factors are abbreviated as Activin A (A), BMP4 (B), and bFGF (F) and their respective concentrations are indicated by the numbers that follow in $\mathrm{ng} / \mathrm{mL}$. To induce PTFla expression, cultures were exposed to $1 \mu \mathrm{g} / \mathrm{mL}$ doxycycline (Dox) renewed every $24 \mathrm{hrs}$ for 3 or 4 days as indicated for the individual experiments. Cells were seeded on two different ECM substrates, Matrigel and Gelatin, for some of the experiments. Methodology for the protocol in Figure 8: Cells were seeded at a density of 60,000 cells $/ \mathrm{cm}^{2}$ in mESC media containing ROCK Inhibitor (Y-27632) either on Gelatin or on Matrigel. Cells were allowed to attach overnight and DE treatment was started the next day. PTFla was induced by adding $1 \mu \mathrm{g} / \mathrm{mL}$ Dox for 4 days overlapping with the end of primitive foregut endoderm stage (PF) and pancreatic endoderm stage (PE).
Following the PE stage, cultures were further treated with Alk5 inhibitor and Nicotinamide (a maturation factor) for 8 days. The source of the growth factors/inhibitors that were used in these experiments is tabulated in Supplementary Table 3 in Supplementary Material available online at http://dx.doi.org/10.1155/2016/6939438.

2.3. Quantitative Real-Time PCR. Cells were harvested at various stages by dissolution and homogenization in $0.5 \mathrm{~mL}$ of Buffer RLT (Qiagen), and RNA was isolated and purified using Qiagen RNAeasy Mini kits. QPCR was performed using Applied Biosystems gene expression assays. Assay IDs are given in Supplementary Table 1. Gapdh was used as an internal control and the comparative threshold method was used to quantify transcript abundance.

2.4. Immunofluorescent Staining. Immunostaining was performed as previously described by Kahan et al. [27]. The antibodies and dilutions are listed in Supplementary Table 2. Secondary antibodies were 488, 568, and 647 Alexa Fluors of anti-goat, anti-mouse, anti-rabbit raised in either goat or donkey. Cells were counterstained with DAPI to mark nuclei. Coverslips with adherent stained cells were mounted on glass slides with Prolong Gold Antifade reagent (Invitrogen). Images were generated using A1R-Si Nikon Confocal or a Zeiss Axiovert 200M microscope.

Immunofluorescent staining was also used to qualitatively measure expression of key transcription factors during differentiation. Flow cytometry was not compatible in many samples involving $3 \mathrm{D}$ structures that were hard to digest to single cells for FACS analysis. Hence, it was decided that costaining and manual counting method would be used to quantify all the samples to maintain consistency. After differentiating mESCs using a multitude of protocols, the cultures were costained for Foxa2 and Sox17 at the definitive endoderm stage and for Pdxl and Nkx6.1 at the pancreatic endoderm stage. Single expressing and coexpressing cells were counted for $N=3$ biological replicates and tabulated in Table 1. Table 1 also indicates seeding densities, format of culture plates, and presence of clustered or scattered expression of markers.

\section{Results}

3.1. Cell Seeding Density Has a Significant Effect on Definitive Endoderm Generation. Our published EB-based differentiation protocol that involves culturing mouse ESCs in suspension as embryoid bodies and plating them in 1\% SR results in a heterogeneous culture that has only some cells coexpressing SOX17 and FOXA2, two markers of DE (Supplementary Figure 1) [24]. To explore the question of PTFla induction in an endoderm context, we pursued generating DE efficiently. Numerous DE-induction protocols were tested using growth factors of the TGF- $\beta$ family, including Nodal, Activin A, and BMP4, and small molecules (Table 1). A universal theme among the various protocols was the impact of cell seeding density on differentiation outcome. Low cell densities promoted DE differentiation irrespective of the protocol or the format of the plate used for cell culture. Adaptation of 


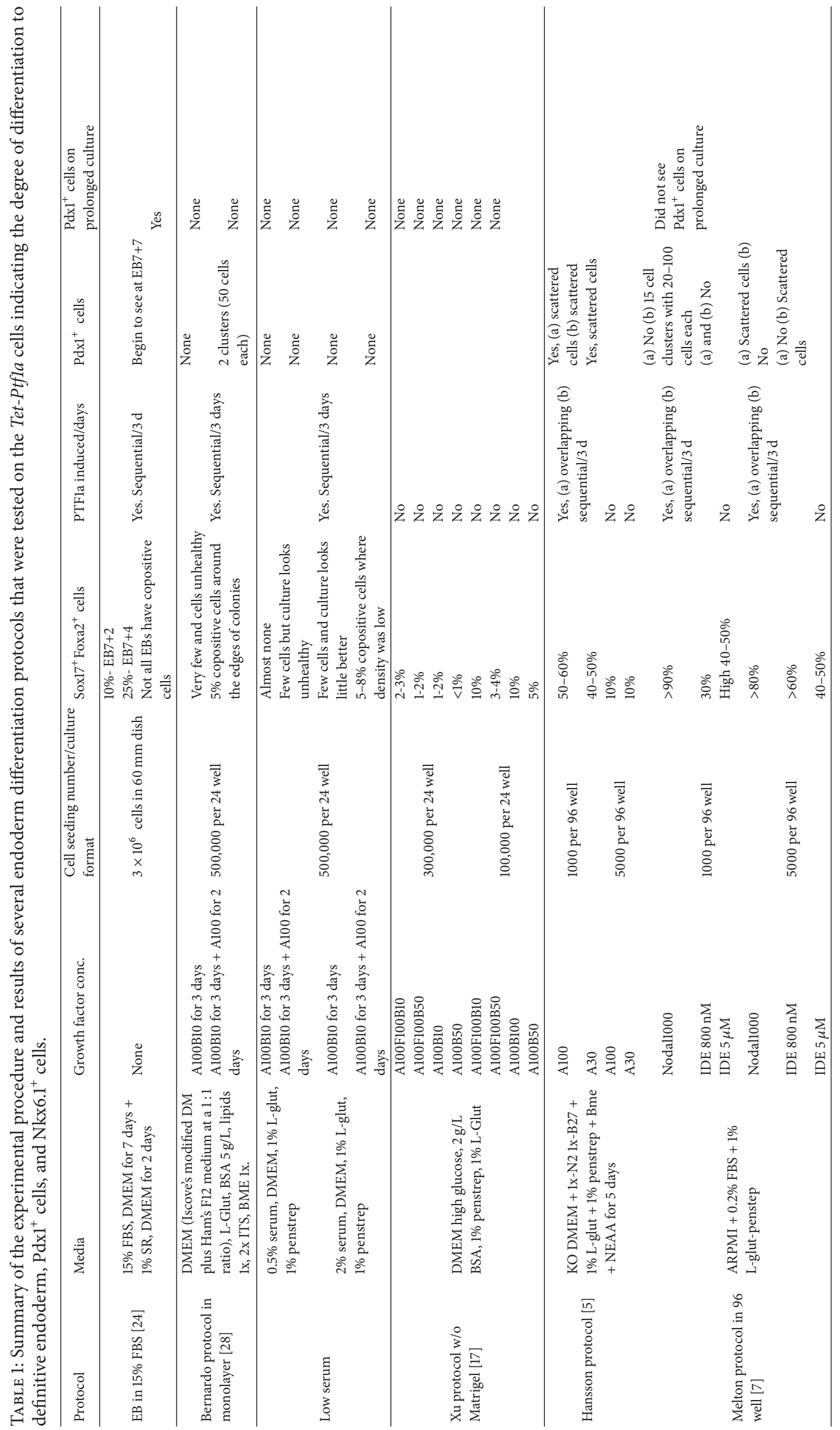




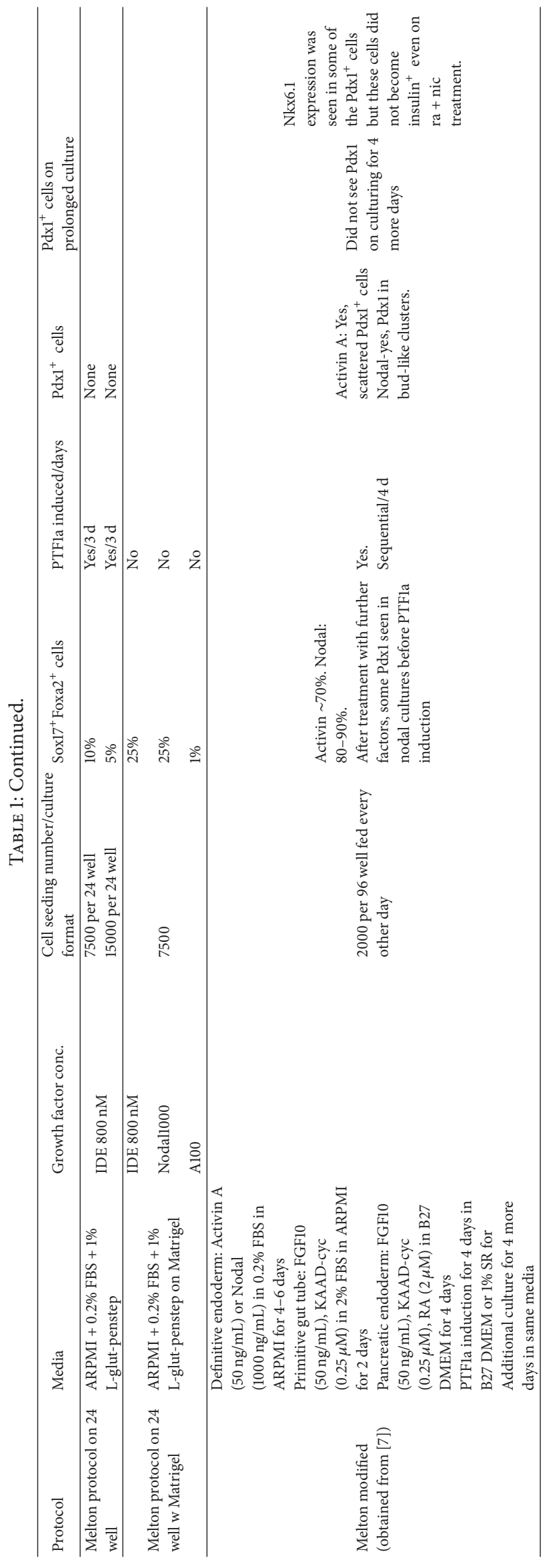


FAB protocol [17], originally devised for hESCs, to mESC differentiation on 24-well plates was more effective in generating DE at an initial cell seeding of 100,000 cells/well versus 300,000 cells/well (corresponding to a cell density of $\sim 50,000$ cells $/ \mathrm{cm}^{2}$ and $\sim 150,000$ cells $/ \mathrm{cm}^{2}$, resp.), as evaluated by Foxa2, T, and Sox17 transcripts, and staining for FOXA2 and SOX17 (Table 1). AB10 (Activin $100 \mathrm{ng} / \mathrm{mL}$ and BMP4 $10 \mathrm{ng} / \mathrm{mL}$ ) and FAB10 (bFGF $100 \mathrm{ng} / \mathrm{mL}$, Activin $100 \mathrm{ng} / \mathrm{mL}$, and BMP4 $10 \mathrm{ng} / \mathrm{mL}$ ) conditions produced significantly more Foxa2, T, and Sox17 transcripts in cultures seeded with 100,000 cells/well than their counterparts seeded with 300,000 cells/well (Figure 1(a)). Similarly, another protocol by Hansson et al. [5] utilizing Activin A in a defined N2B27 medium was more successful in 96-well plates at a lower cell density of 1000 cells/well $\left(\sim 3000\right.$ cells $\left./ \mathrm{cm}^{2}\right)$ compared with 5000 cells/well $\left(15000\right.$ cells $\left./ \mathrm{cm}^{2}\right)$. This pattern was, again, observed for two different concentrations of Activin A (Figure 1(b)). Immunofluorescent costaining for FOXA2 and SOX17 was consistent with the transcript profile showing that cultures with low starting cell density formed more DE than high density cultures. Moreover, $\mathrm{SOX}^{+}{ }^{+} \mathrm{FOXA}^{+}$cells were always observed at the edges of the colonies (Figure 1(c)) possibly suggesting that $\mathrm{DE}$ formation requires less cell-cell contact.

\subsection{Inducing PTF1a in Monolayer Endodermal-Derived Cells} Generated Pdx1 Nkx6.1 $1^{+}$Pancreatic Progenitors. Achieving DE differentiation from mESC monolayer cultures has been challenging with low yields and poor cell survival. Tet-Pt1a mESCs were subjected to several DE differentiation protocols that were published over the course of several years. Initial protocols that were tested had very low efficiency of forming SoX $17^{+} \mathrm{FOXA}_{2}{ }^{+}$cells. Subsequent protocols made modifications to the basal media and additives and/or concentration of growth factors and reported improved DE formation [5, 17], and these also led to robust DE generation by the Tet-Ptfla cells. The results of these protocols are summarized in Table 1. Treatment with Activin A, Nodal, and IDE1 gave rise to DE as marked by SOX $17^{+} \mathrm{FOXA}^{+}$cells (Supplementary Figure 1) and $\mathrm{ECADH}^{+} \mathrm{FOXA}_{2}{ }^{+}$cells and some $\mathrm{ECADH}^{-} \mathrm{FOXA}_{2}{ }^{+}$ mesodermal cells (Supplementary Figure 2) at differing frequencies. Nodal was the most effective in converting the majority of cells to $\mathrm{DE}$ as indicated by the number of $\mathrm{SOX}_{1}{ }^{+} \mathrm{FOXA}_{2}{ }^{+}$and $\mathrm{ECADH}^{+} \mathrm{FOXA}_{2}{ }^{+}$cells. Ectopic PTFla expression was induced in DE obtained from many of these protocols to test the hypothesis that PTFla activity triggers the pancreatic developmental program more efficiently in an endoderm enriched population compared to spontaneously differentiated EB cultures and ultimately leads to enhanced pancreatic differentiation. Pdxl activation was designated as the first landmark of pancreatic differentiation, in particular pancreas specification of the naïve endoderm. A short PTF1a induction for 3 days ( $1 \mu \mathrm{g} / \mathrm{mL}$ Dox addition), either sequentially following $\mathrm{DE}$ generation or overlapping with the last 2 days of DE generation, was performed. PDX1 expression was evaluated shortly after (ranging from 3 to 4 days) PTFla induction. Scattered $\mathrm{Pdxl}^{+}$cells were found in cultures that were grown using the Hansson protocol [5], whereas nodalgenerated $\mathrm{DE}$ [7] produced $\mathrm{Pdxl}^{+}$bud-like clusters after sequential PTFla induction (data not shown). However, when these cultures were analyzed a week later, PDX1 expression was absent (results compiled in Table 1). These observations suggested that unpatterned $\mathrm{SOX} 17^{+} \mathrm{FOXA}^{+} \mathrm{DE}$ population does not have the right cellular context to respond to PTFla activity.

Hence, we pursued further differentiation of naïve endoderm into primitive gut (PG) and posterior foregut endoderm (PF) (modified Melton protocol) to establish the right cell types before inducing PTFla. Activin A $(50 \mathrm{ng} / \mathrm{mL})$ or Nodal $(1000 \mathrm{ng} / \mathrm{mL})$ in low serum media was used to generate endoderm. DE markers, Sox17 and Foxa2, were significantly upregulated in both Activin A- and Nodal-DE compared to control cultures (cells that did not receive growth factors but were cultured in the same base media indicating spontaneous differentiation) (Figure 2(a)). Activin A- and Nodal-DE had more $\mathrm{SOX}_{1}{ }^{+} \mathrm{FOXA}^{+}$cells than control cultures, and the control cultures also had large numbers of $\mathrm{SOX}^{-} 7^{-} \mathrm{FOXA}^{+}$ mesodermal cells (Figure 2(b)). As these two transcription factors are also present in extraembryonic endoderm (EE), it is important to determine the expression of Sox7, a marker of EE. Notably, Sox7 expression was reduced in DE cultures. In addition, Sox1, a regulator of ectoderm lineage, and Meoxl, an indicator of mesoderm differentiation, were significantly lower in Activin A- and Nodal-DE than in controls (Figure 2(a)). After subsequent differentiation to PG and PF, cultures were analyzed for markers of PF. There were significantly higher levels of $H l x b 9$ and $H n f 6$ transcripts in Activin A-PF $(P<0.05$ and $P<0.001$, resp. $)$ and NodalPF $(P<0.01$ and $P<0.001$, resp. $)$ compared to control cultures that did not receive any growth factors. Hnf4a, a liver progenitor maker, was downregulated in both Activin A-PF and Nodal-PF cultures indicating that they have been specified to the pancreatic lineage $(P<0.01)$. HnflB and $P d x 1$ were significantly elevated in the Nodal cultures $(P<$ 0.05) (Figure 3(a)). Immunofluorescent staining of Nodal-PF cultures confirms the gene expression profile (Figure 3(b)). $\mathrm{PDXl}^{+}$areas, however, were very few and typically appeared as a subset of $\mathrm{HNF}^{+}$domains (Figure 3(b)).

FGF10 is involved in the proliferation of pancreatic progenitors $[29,30]$ and in the maintenance of PTFla expression in the dorsal pancreatic bud [31]. Therefore, we treated the Activin A-PF and Nodal-PF with FGF10 during the induction of PTFla. PTFla induction in both Activin A-PF and Nodal$\mathrm{PF}$ cultures resulted in many $\mathrm{PDX1}^{+} \mathrm{NKX} 6.1^{+}$areas. For example, larger domains of PDX1 expression containing small clusters of NKX6.1 $1^{+}$cells can be seen (Figure 4(a)). Nkx6.1 is initially expressed in the pancreatic epithelium, but it is a marker of the trunk domain that is poised to become endocrine/duct lineage at later stages. Next, we wanted to investigate whether the formation of pancreatic epithelium was specific to the PTFla-induced cultures or was caused by FGF10. Nodal-PF cultures that were induced with Dox but not treated with FGF10 also gave rise to $\mathrm{PDX1}^{+} \mathrm{NKX} 6.1^{+}$ cells, though lower in number than combined treatment (Figure 4(b), top row). More importantly, Nodal-PF cultures treated with FGF10 alone did not produce any NKX6.1 ${ }^{+}$ 


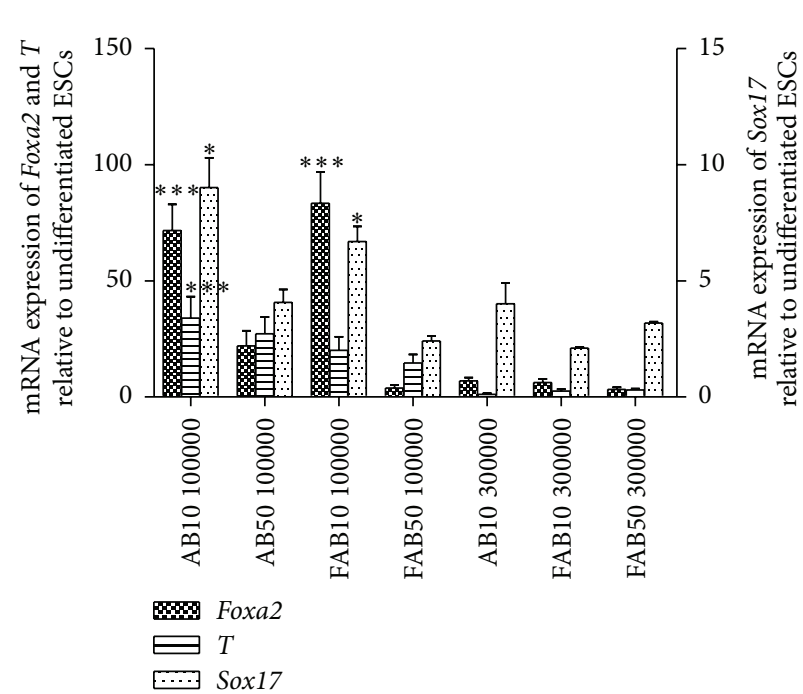

(a)
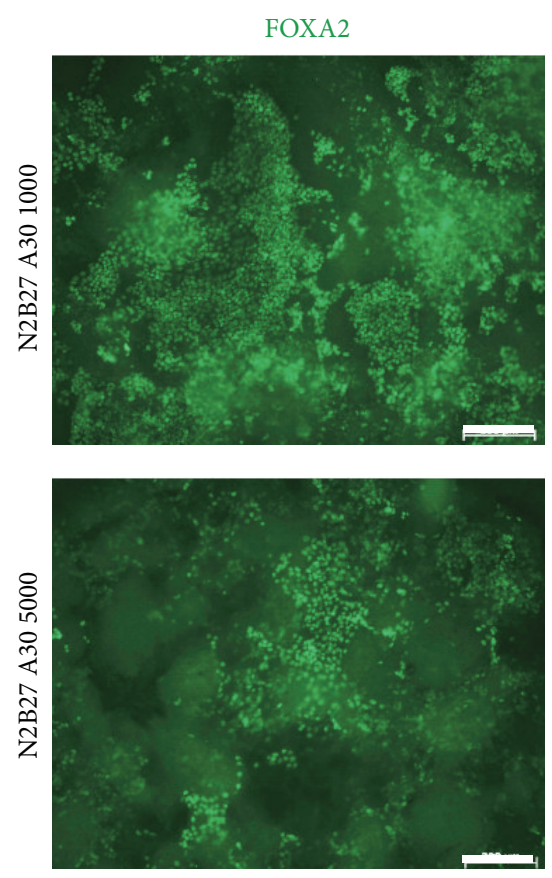

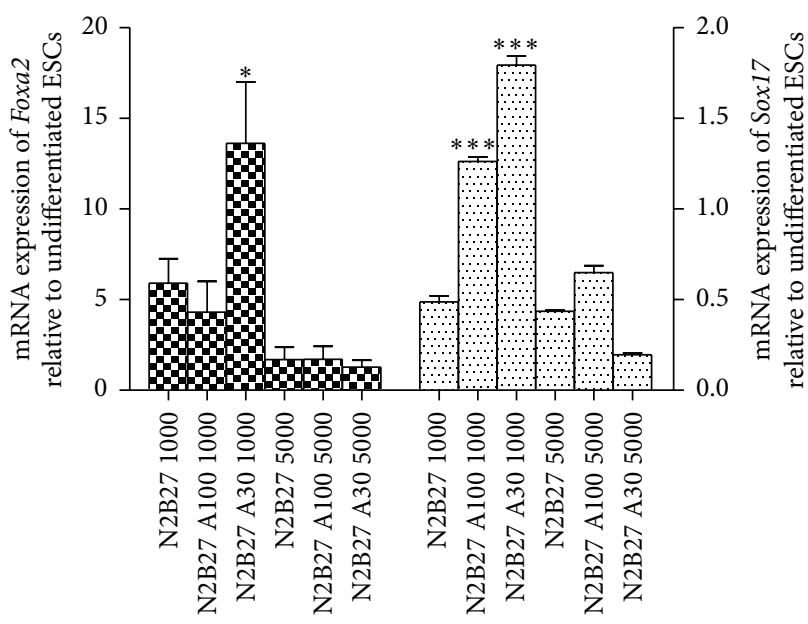

Eoxa2 Sox 17

(b)
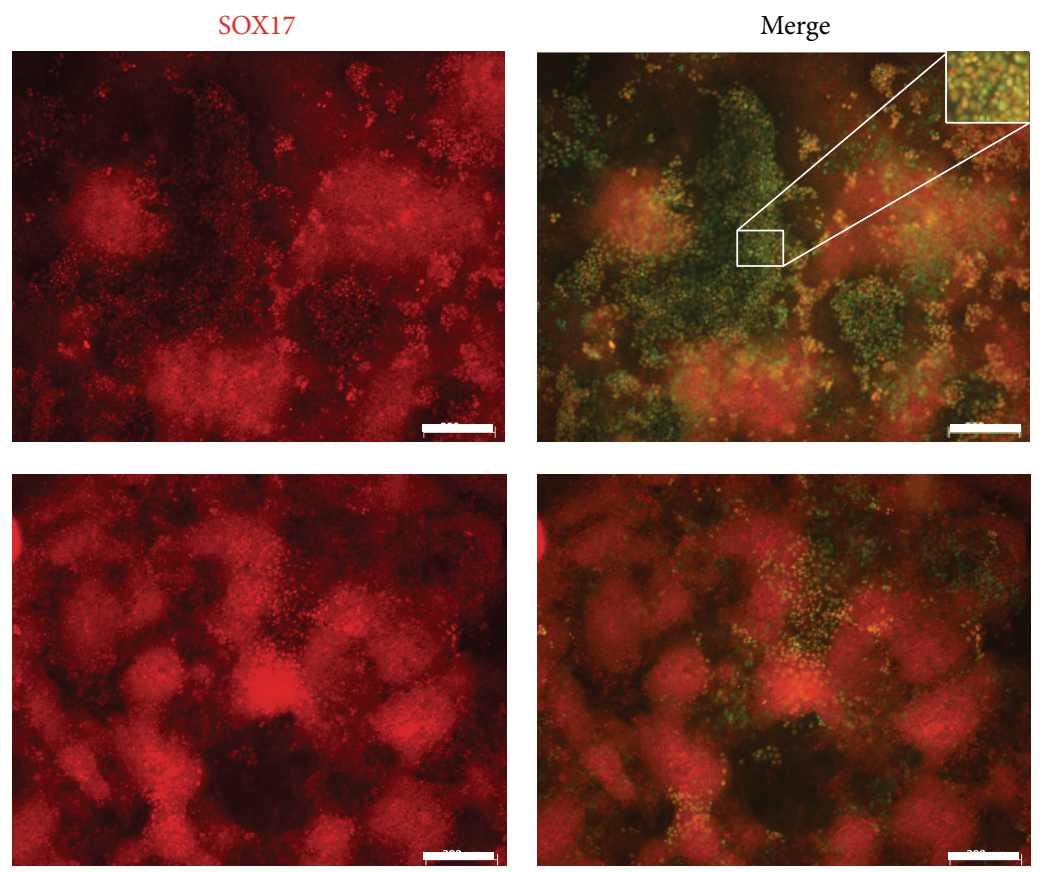

(c)

FIGURE 1: Cell seeding density influences definitive endoderm generation. (a) Cultures seeded with two different mESC densities (100,000 cells/well or 300,000 cells/well of a 24 -well plate) were subjected to a 3-day endoderm differentiation protocol containing $100 \mathrm{ng} / \mathrm{mL}$ bFGF, $100 \mathrm{ng} / \mathrm{mL}$ Activin A, and $10 \mathrm{ng} / \mathrm{mL}$ or $50 \mathrm{ng} / \mathrm{mL}$ of BMP4. (b) Cells cultured using another endoderm protocol containing Activin A $100 \mathrm{ng} / \mathrm{mL}$ or $30 \mathrm{ng} / \mathrm{mL}$ for 5 days in N2B27 but seeded with two starting cell densities (1000 cells/well or 5,000 cells/well of a 96 well plate). Relative mRNA expression of Sox17, $T$, and Foxa2 indicates low densities led to higher expression of DE markers. $N=2$. Data are presented as mean \pm SEM. Asterisks indicate $P$ values on comparison with corresponding high density cultures: ${ }^{*} P<0.05,{ }^{* *} P<0.01$, and ${ }^{* * *} P<0.001$ determined by one-way ANOVA with Tukey's multiple comparison test. (c) Immunofluorescent costaining for FOXA2 and SOX17 confirms that cultures that started with low cell density (N2B27 A30 1000) had higher numbers of FOXA2 ${ }^{+} \mathrm{SOX}^{+} 7^{+}$definitive endoderm than cultures seeded with high density (N2B27 A30 5000). Images at 5x. Higher magnification inset to indicate coexpression at the cellular level. Scale bars, $200 \mu \mathrm{m}$.

cells (Figure 4(b), second row). These results suggest that PTFla induction is important to generate a true pancreatic epithelial progenitor phenotype $\left(\mathrm{PDX1}^{+} \mathrm{NKX} 6.1^{+}\right)$, and that it acts synergistically with FGF10. Above all, it is to be noted that inducing PTFla in the non-Nodal-PF cultures (i.e., not endodermal enriched cultures) resulted in a few small isolated $\mathrm{PDX1}^{+}$clusters and wide-spread scattered NKX6.1 expression (Figure 4(b), third row) with the $\mathrm{PDX1}^{+}$cells expressing NKX6.1 but with many NKX6.1 ${ }^{+} \mathrm{PDX1}^{-}$cells as well. These NKX6.1 $1^{+} \mathrm{PDX}^{-}$cells are not pancreatic and could 

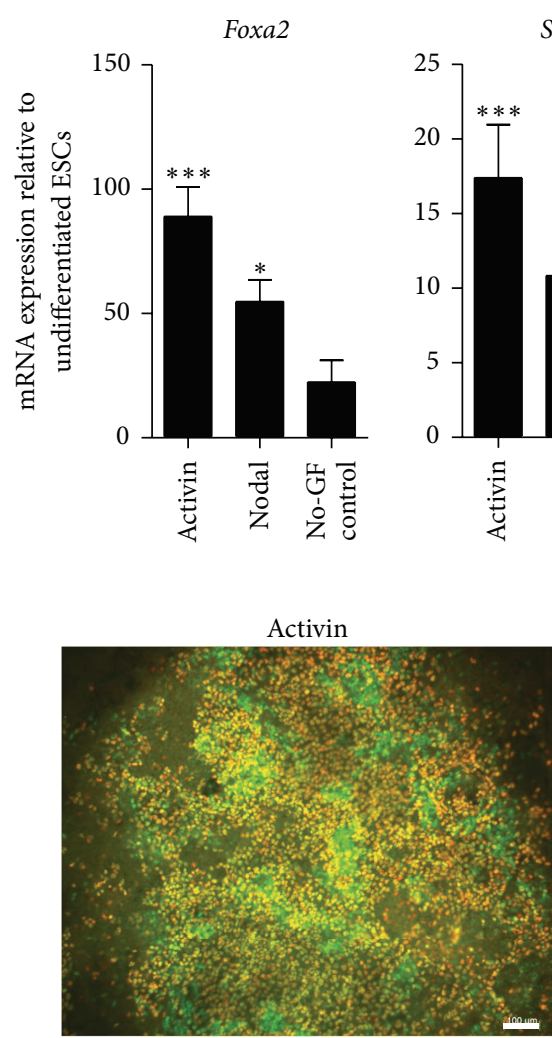

Sox17
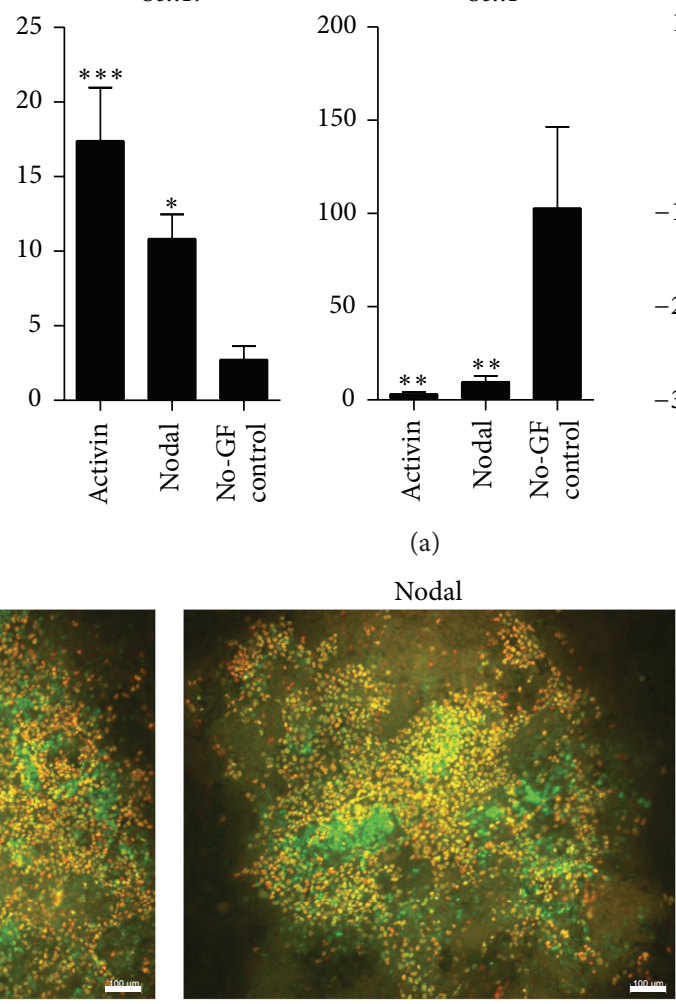

FOXA2/SOX17/Merge
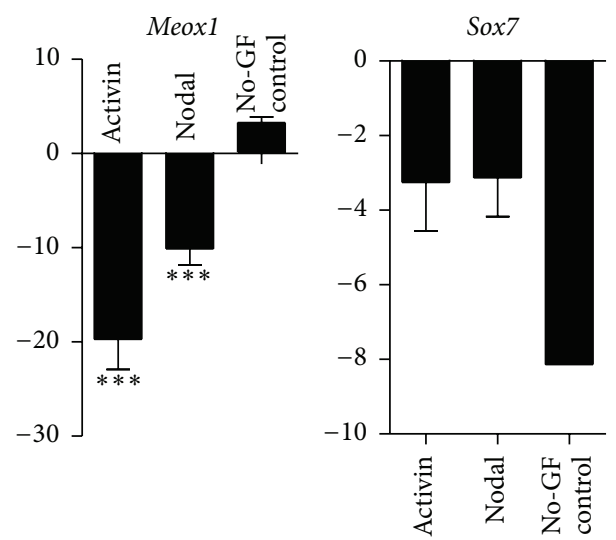

(b)

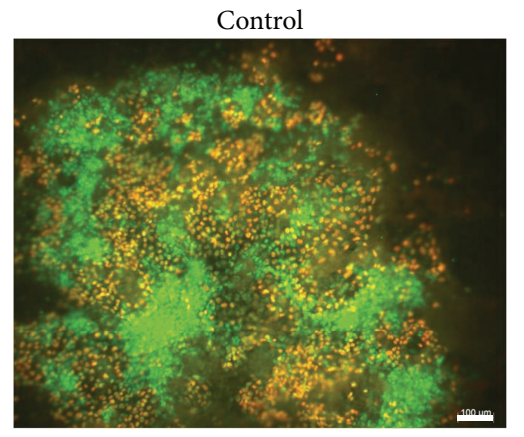

FIgURE 2: Markers of DE are highly expressed in Nodal and Activin A treated cells, whereas markers of other lineages, including ectoderm, mesoderm, and extraembryonic endoderm are repressed. (a) DE markers, Sox17 and Foxa2, are upregulated in contrast to the genes of other germ layers, ectoderm (Sox1), mesoderm (Meoxl), and extraembryonic endoderm (Sox7) in Nodal and Activin A treated cultures. $N=3$. Data are presented as mean \pm SEM. Asterisks indicate $P$ values on comparison with no-growth factor treated (No-GF) control cultures: ${ }^{*} P<0.05$, ${ }^{* *} P<0.01$, and ${ }^{* * *} P<0.001$ determined by one-way ANOVA with Tukey's multiple comparison test. (b) Activin A and Nodal treatment lead to substantial number of FOXA2 ${ }^{+} \mathrm{SOX} 7^{+}$cells, whereas the No-GF control cultures generate FOXA2 ${ }^{+} \mathrm{SOX}_{17^{-}}$populations. Scale bars, $100 \mu \mathrm{m}$.

potentially be of neural lineage, as shown by elevated levels of Soxl in control (no growth factor treated) cultures early in differentiation (Figure 2(a)). In other words, PTFla activity in an endodermal context, specifically in prepatterned posterior foregut endoderm, improves the formation of pancreatic progenitor cells compared to induction in spontaneously differentiated cultures.

\subsection{Differentiation to Adult Pancreatic Cell Types Is Enhanced} by $3 D$ Culturing in the $D E / P E$ Stage. On further differentiation of monolayer cultures of PTFla-induced Activin A/Nodal-PF, we did not observe a concomitant increase in terminally differentiated pancreatic cell types, including insulin $^{+}$, glucagon ${ }^{+}$, somatostatin $^{+}$, or amylase $^{+}$cells; in fact, only rare insulin ${ }^{+}$cells were found. Monolayer cultures, though homogenous, lack complex morphogenesis and paracrine signaling present in $3 \mathrm{D}$ cultures $[32,33]$ and hence may not be the optimal way to direct differentiation efficiently into adult cell types. While testing monolayer differentiations on two different ECM substrates, Gelatin or Matrigel, using a protocol adapted from Sneddon et al. [25] (Figure 5), we observed an interesting phenomenon. Floating EB-like subpopulations emerged in both DE and PG stages from Gelatin-coated dishes. These spheres arose only from Gelatincoated dishes possibly due to differences in integrin signaling mediating cell-ECM interactions on those two substrates. It has been shown that E-cadherin is more stable in cells on Matrigel than on Gelatin resulting in more stable attachment to Matrigel [34]. Serendipitously, the floating spheres were collected, replated, and differentiated as per the protocol till the end stage.

It is to be noted that the two ECMs differed in their propensities to generate DE: cells seeded on Gelatin expressed significantly more Foxa2 than ones seeded on Matrigel, whereas Sox17 expression was comparable between the two groups (Supplementary Figure 3). PTFla was induced for 4 days overlapping with the end of PF stage and the beginning of pancreatic endoderm (PE) differentiation as shown in Figure 5. Cells were further cultured for 8 days in Alk5 inhibitor and Nicotinamide, and terminal differentiation to adult pancreatic cell types was assessed. Transcript profiles of endocrine hormones, namely, Insulin, Gcg and 


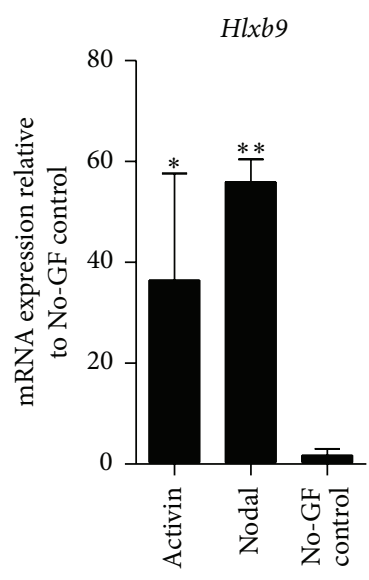

PDX1
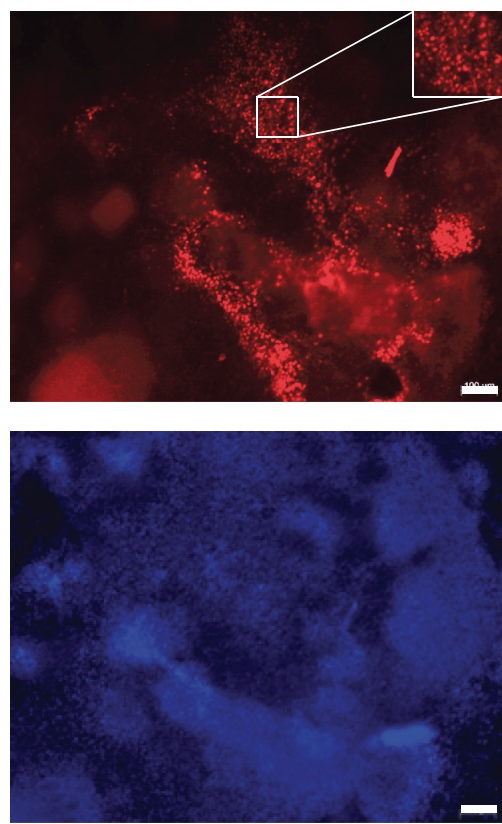

DAPI
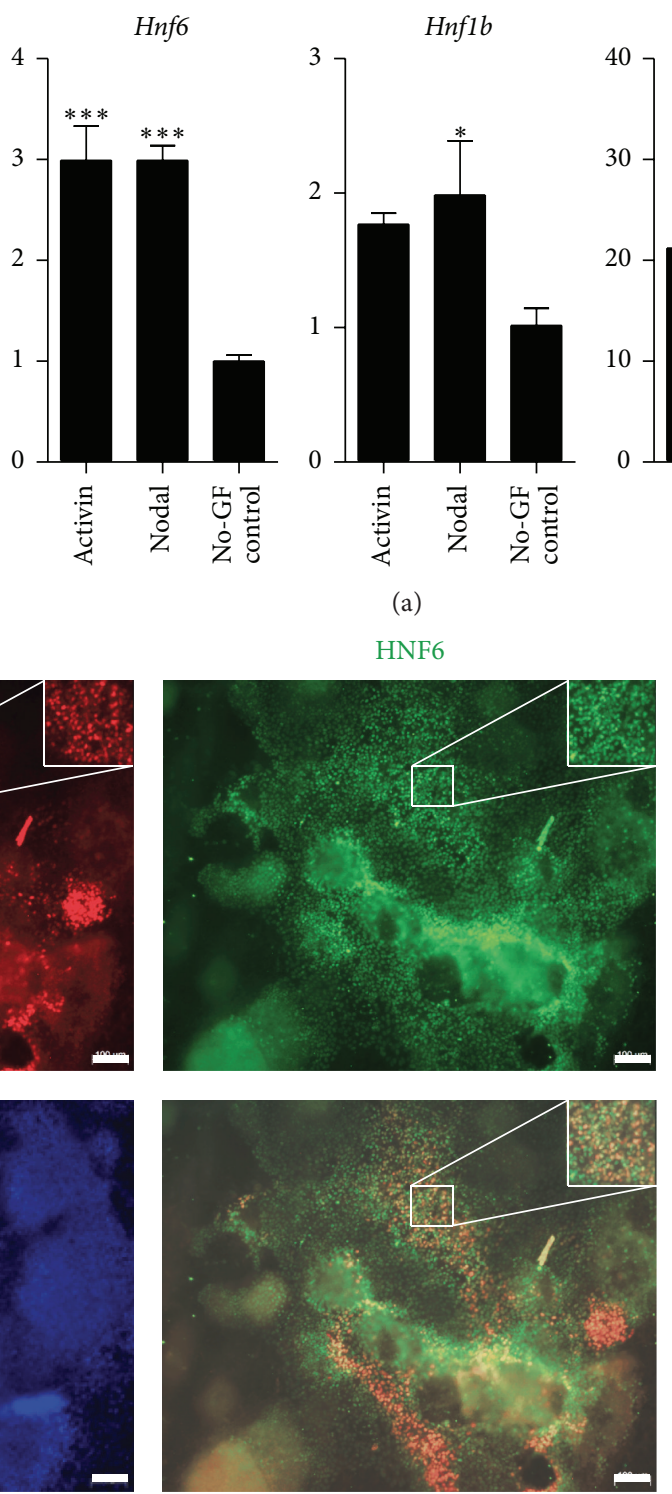

Merge
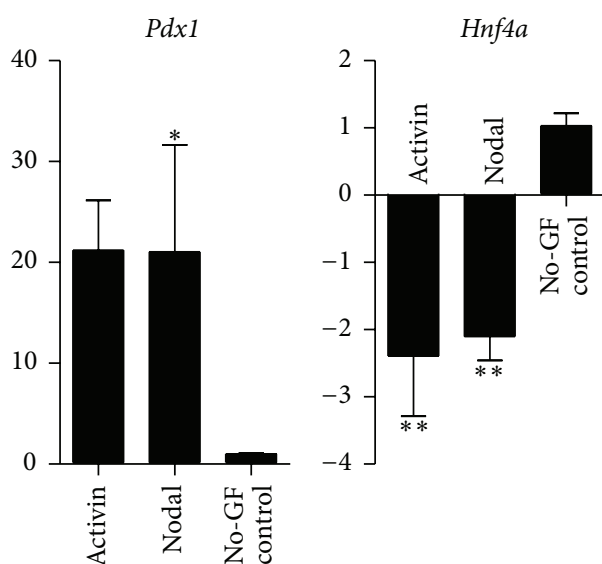

HLXB9
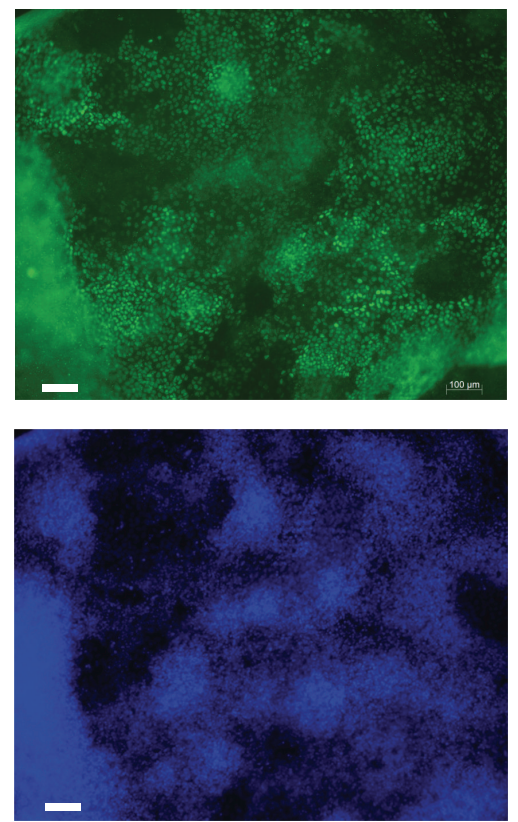

DAPI

(b)

Figure 3: Analysis of mESC-derived posterior foregut endoderm. (a) Posterior foregut endoderm markers, $H l x b 9, H n f 6, H n f 1 b$, and $P d x 1$, were significantly higher in Nodal- and Activin-derived cultures than in no growth factor treated (No-GF) control cultures. On the other hand, Hnf $4 a$ that is expressed in liver progenitors was reduced. $N=2-3$. Data are presented as mean \pm SEM. Asterisks indicate $P$ values on comparison with No-GF control cultures: ${ }^{*} P<0.05,{ }^{* *} P<0.01$, and ${ }^{* * *} P<0.001$ determined by one-way ANOVA with Tukey's multiple comparison test. (b) Immunofluorescent staining of Nodal-derived posterior foregut endoderm cultures indicates the expression of the above-mentioned markers at the single-cell protein level. Pdxl is expressed in Hnf6-expressing cells. Scale bars, $100 \mu \mathrm{m}$.

Sst, and exocrine digestive enzyme, $A m y 2 a$, were analyzed in the different cultures. Upon induction of PTFla, subpopulations that were in suspension (floating) in DE or PG stages (Suspension-DE/Suspension-PG) expressed more endocrine- and exocrine-specific genes than those that were differentiated throughout in adherent cultures (Figure 6). Suspension-DE group had the maximum level of Insulin, $\mathrm{Gcg}$, and Amy2a, whereas Suspension-PE group had maximum Sst expression (Figure 6). Cultures that were in suspension for a brief period of time responded better to PTFla activity and showed enhanced terminal differentiation. Among the adherent cultures, we found that Gelatin performed better than Matrigel as an ECM substrate in the differentiation of Dox-treated cells as judged by significantly higher expression of Amy2a, Gcg, Sst, and Insulin. Matrigel, however, seemed to promote the expression of endocrine transcripts in uninduced cultures (Supplementary Figure 4).

In concert with gene expression, staining for amylase- and hormone-expressing cells (antibody cocktail against insulin, glucagon, and somatostatin) also revealed extensive differentiation of PTFla-induced Suspension-DE/Suspension-PG cultures to both endocrine and acinar lineage (Figure 7, 

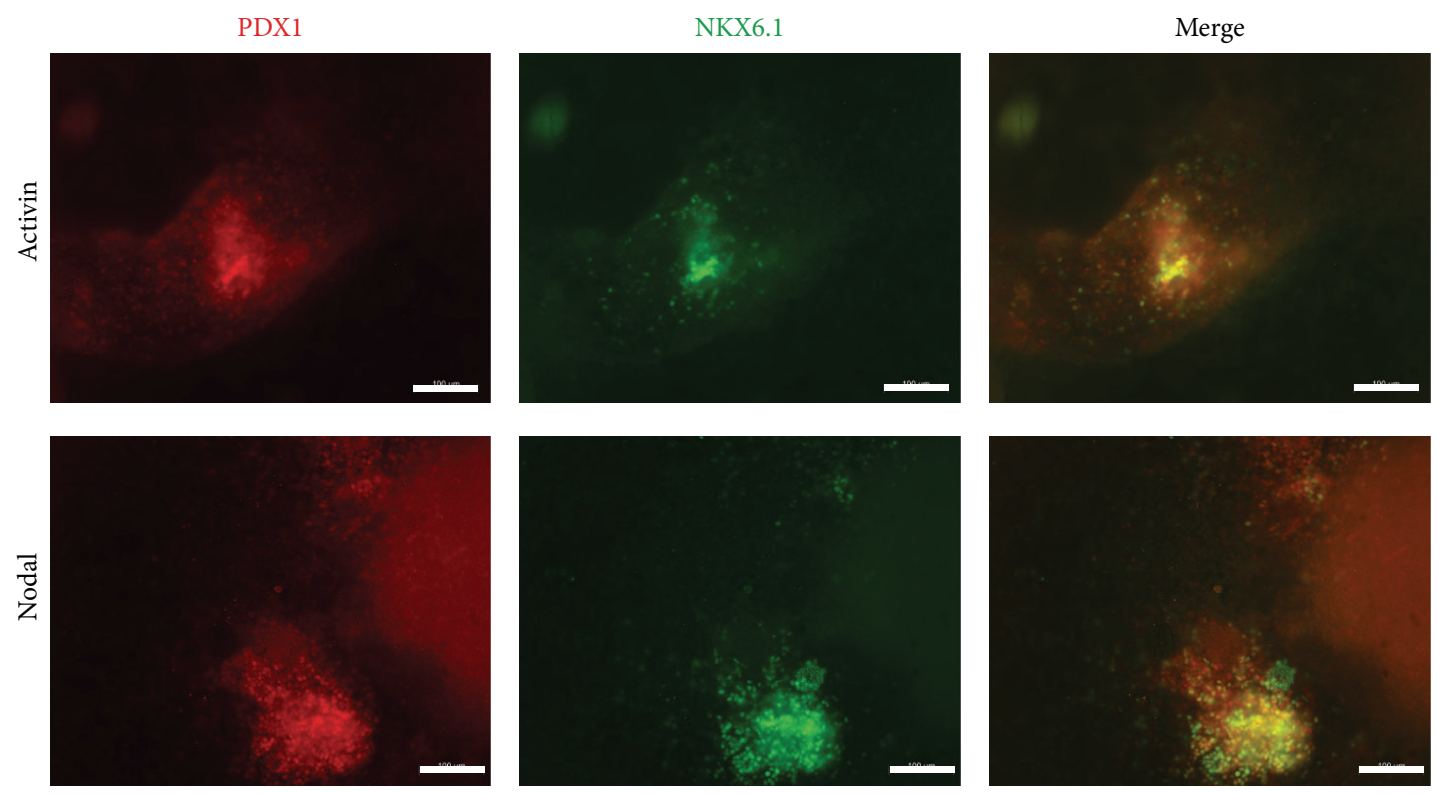

(a)
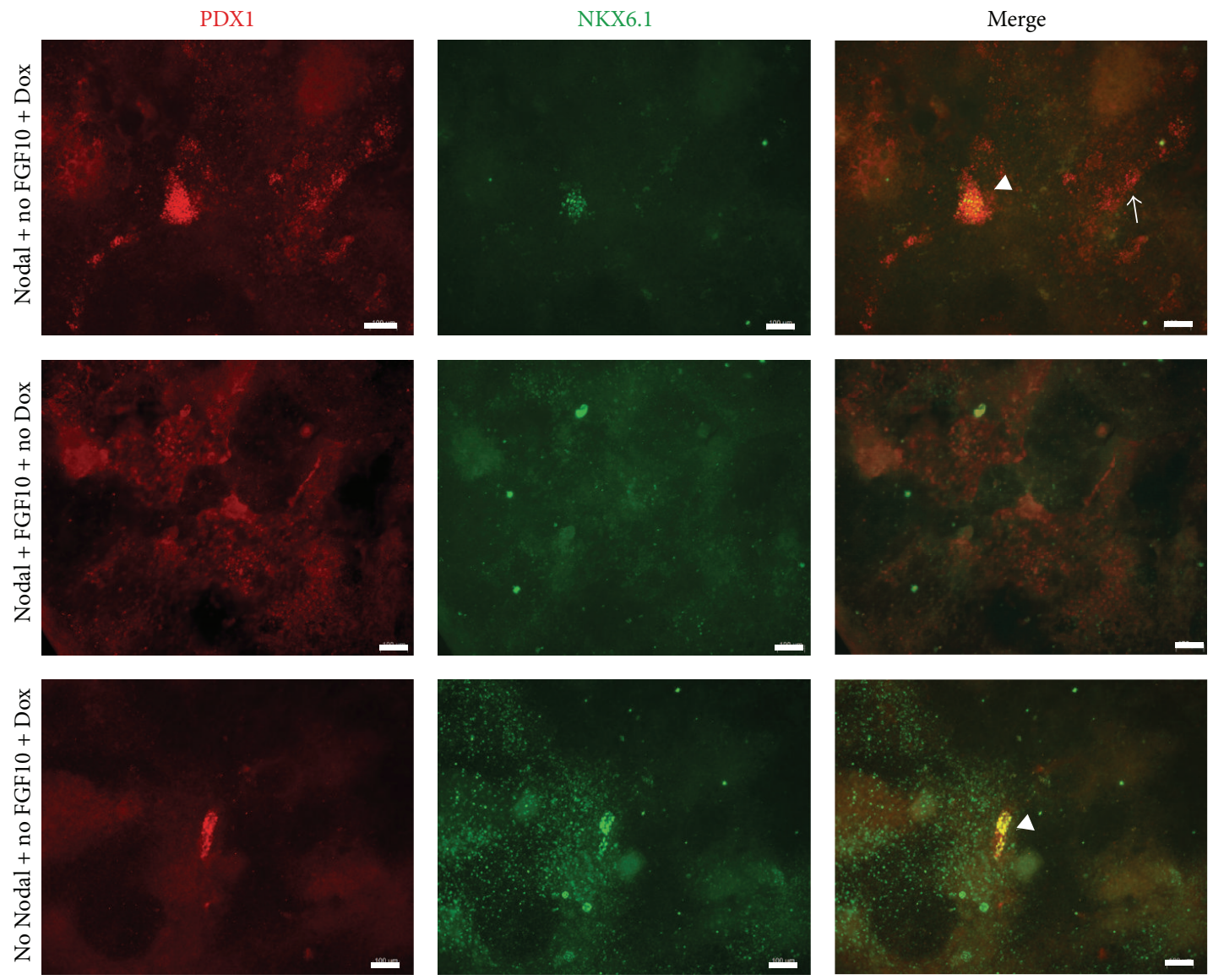

(b)

FIgURE 4: PTFla induction in combination with FGF10 led to the formation of pancreatic epithelial progenitors. (a) Both Nodal and Activin A derived posterior foregut endoderm generated PDX1 ${ }^{+}$NKX6.1 $1^{+}$pancreatic progenitors on induction of PTFla along with FGF10 treatment. Uninduced cultures did not have any such copositive area (data not shown). (b) Nkx6.1 and Pdx1 copositive pancreatic progenitors were unique to PTFla induced cultures (top and bottom row). FGF10 was not necessary to generate PDX1 ${ }^{+} \mathrm{NKX} 6.1^{+}$population, although it increased the numbers of such progenitors (top row). Notably, FGF10 alone did not produce any NKX6.1 $1^{+}$cells (middle row), and hence PTFla induction is essential for the formation of this pancreatic progenitor population. Arrowheads indicate PDX1 and NKX6.1 double positive costained population. Arrows indicate PDX1 single positive cells. Scale bars, $100 \mu \mathrm{m}$. 


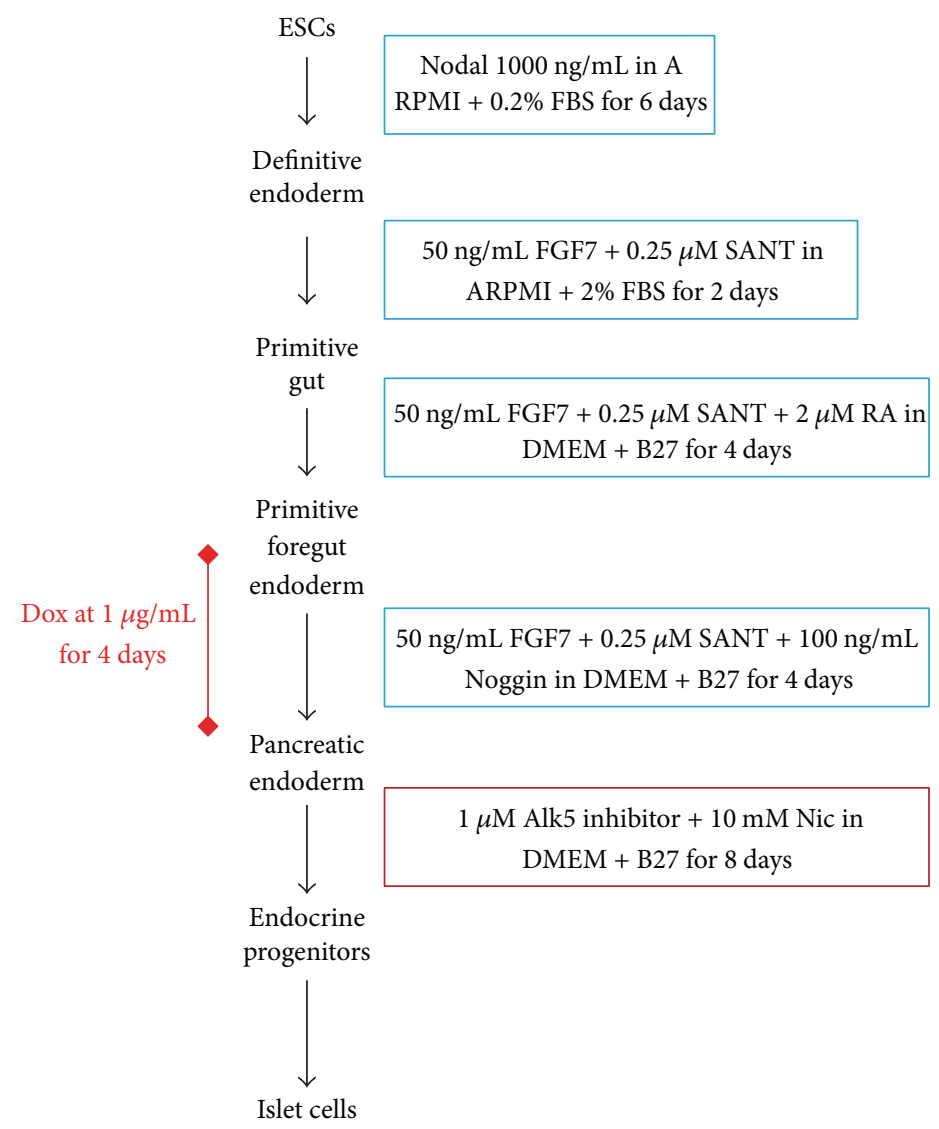

Figure 5: Pancreatic differentiation protocol adapted from Sneddon et al. [25]. Cells were seeded at a density of $60,000 \mathrm{cells} / \mathrm{cm}^{2}$ either on Gelatin or on Matrigel. PTFla was induced by adding $1 \mu \mathrm{g} / \mathrm{mL}$ Dox for 4 days overlapping with the end of primitive foregut endoderm stage (PF) and pancreatic endoderm stage (PE). Following the PE stage, cultures were further treated with Alk5 inhibitor and Nicotinamide (a maturation factor) for 8 days and analyzed for markers specific to adult pancreatic cell types.

middle and lower rows). Qualitatively, it appeared that the Suspension-DE cultures produced more endocrine and acinar cells than Suspension-PG cultures. High magnification images of Suspension-DE cultures demonstrated that the amylase $^{+}$and the hormone ${ }^{+}$cells were distinct (Figures 8(a) and $8(\mathrm{~b})$ ) and that the insulin ${ }^{+}$cells in the cultures do not express glucagon (Figure 8(c)), while most express nuclear PDX1 (Figure 8(d)).

There was another difference in the way the suspension and the adherent cultures were treated. To enable reattachment, the floating EB-like population was plated in 15\% FBS overnight and subjected to subsequent steps in the differentiation protocol as usual. Thus, to rule out that transient serum exposure might be the cause for enhanced pancreatic differentiation, a control experiment was performed where the adherent cultures received overnight serum-bolus mimicking the period of attachment of the floaters. mRNA expression analysis from these cultures (adherent DE/PG) did not show comparable expression of Amy $2 a$, insulin, $\mathrm{Gcg}$, or Sst transcripts as the cultures that underwent suspension culturing (Supplementary Figure 5) and immunofluorescent staining (data not shown) confirmed these results, suggesting that the physical state of suspension was responsible for the enhanced pancreatic differentiation that we observed.

\section{Discussion}

The pancreas is an organ of endodermal origin, arising from a narrow Shh signaling-excluded region in the posterior foregut endoderm. Therefore, the context of expression of key pancreatic transcription factors (PDX1 and PTFla) involved in pancreas specification is extremely unique and is important for the successful activation of the pancreatic program. Previously we have shown that ectopic expression of PTFla in EB-based cultures leads to the formation of all pancreatic lineages: endocrine, acinar, and duct cells. In particular, in vitro cultures recapitulated major morphological and molecular events that occur during pancreas organogenesis. In these studies, we hypothesized that induction of PTFla in an enriched endodermal context would lead to better pancreatic differentiation and that PTFla could potentially be the missing element in the current $\beta$ cell differentiation protocols that fail to generate monohormonal insulin ${ }^{+}$cells.

To this end, we generated DE using numerous published protocols as illustrated in Table 1. Many of these protocols showed abysmal performance with low efficiency and/or low cell viability. In contrast to the efficient differentiation of hESC to DE in monolayers using high Activin A and low serum supplementation, mESCs have proven to be rather 


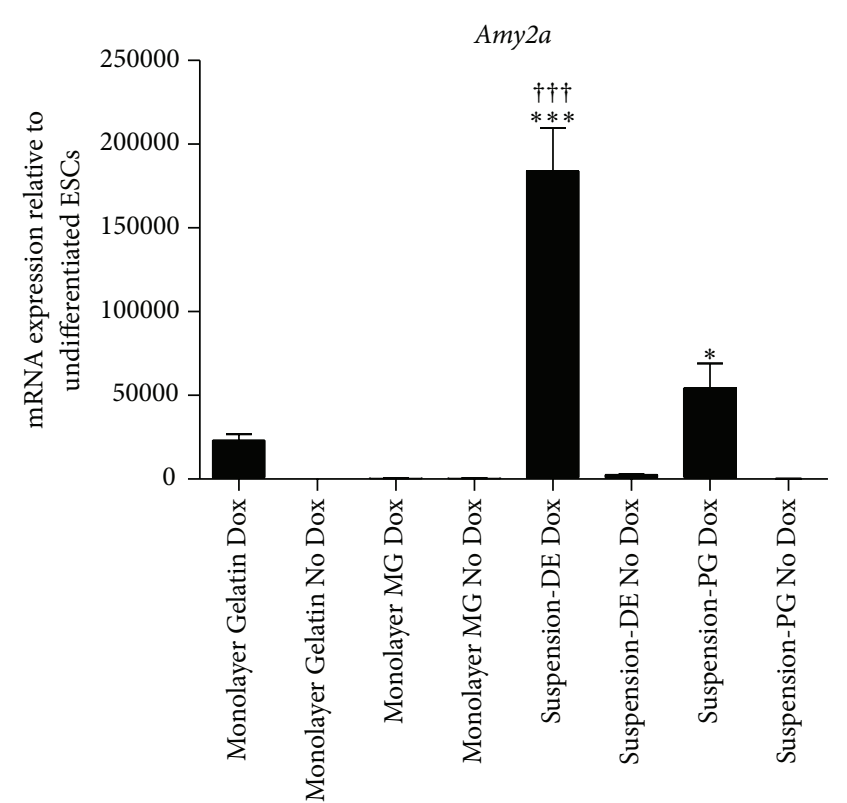

(a)

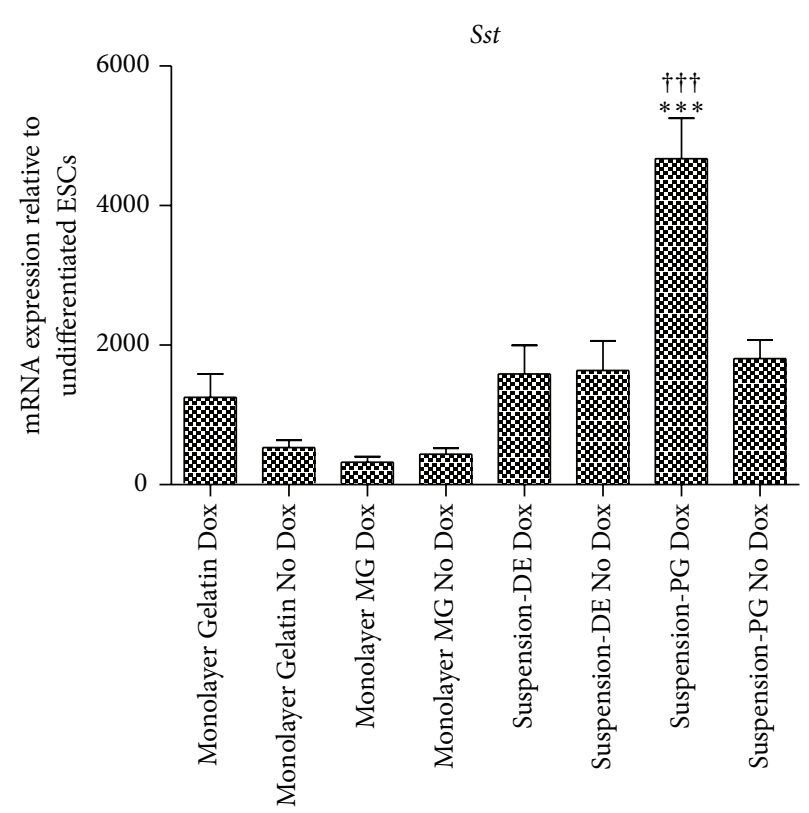

(c)

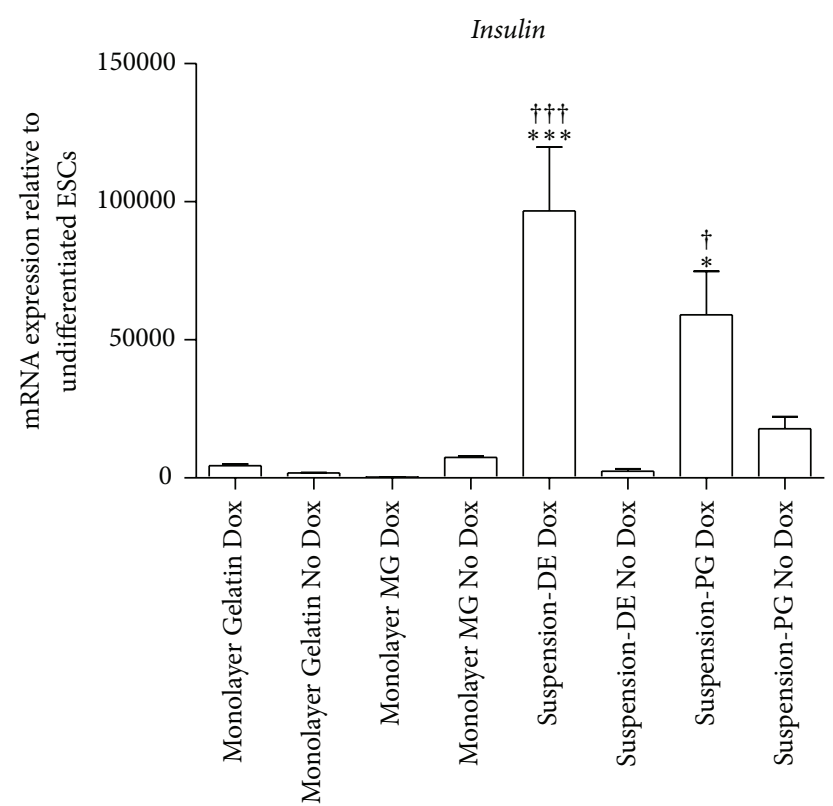

(b)

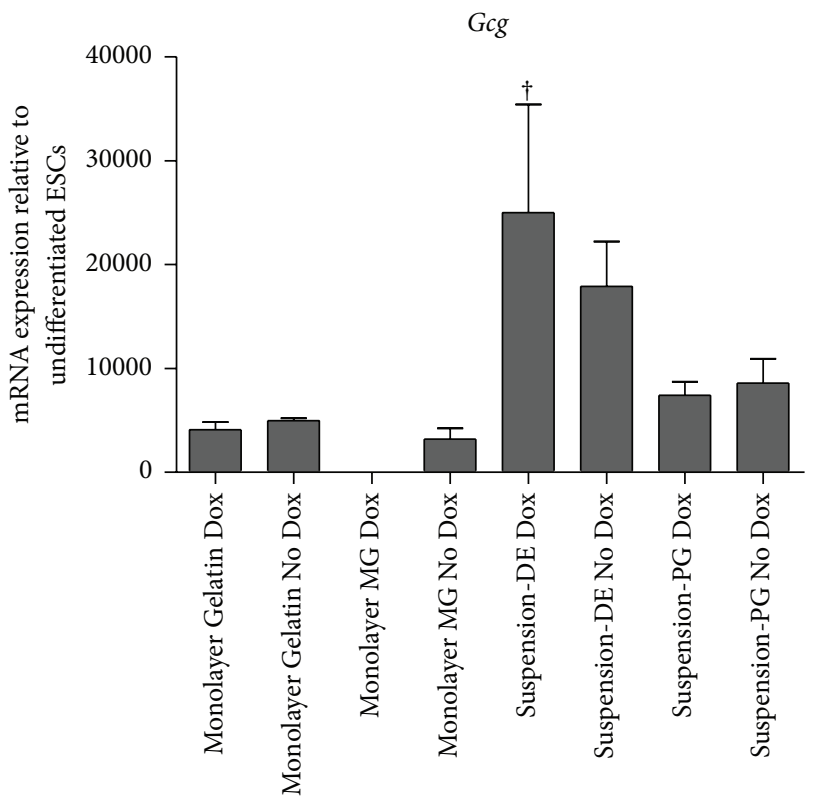

(d)

Figure 6: A short 3D culture period at the beginning of differentiation promotes PTFla-induced pancreatic differentiation. Populations that formed EB-like floating bodies either in DE or in PG stage, when replated and subjected to the differentiation protocol, expressed significantly more Amy2a (a), Insulin (b), Sst (c), and Gcg (d) transcripts than those cultures grown as monolayers throughout. Growing in suspension also improved endocrine differentiation in general (Suspension-DE No dox and Suspension-PG No dox) indicated by elevated Insulin, Sst, and $G c g$ transcripts. $N=3$. Data are presented as mean \pm SEM. Asterisks indicate $P$ values on comparison with Gelatin-No Dox cultures: ${ }^{*} P<0.05,{ }^{* * *} P<0.001$, and Obelisks indicate $P$ values on comparison with Gelatin-Dox cultures: ${ }^{\dagger} P<0.05$ and ${ }^{\dagger \dagger \dagger} P<0.001$ determined by one-way ANOVA with Tukey's multiple comparison test.

difficult $[1,3-5,16]$. During our various trials, we observed a common trend: cell seeding density influenced the success of endoderm generation. Cultures that started with low cell densities differentiated to DE more readily than ones with high densities. Our findings correlate well with similar observations that low cell densities promoted RA-induced PDX1 expression in hESCs in culture [35], where cell-cell contact was found to be inhibitory for pancreatic differentiation. It has also been shown that mESCs grown at high densities have their $\beta$-catenin pool localized at the plasma membrane [36] and membrane association of $\beta$-catenin with Oct4/Ecadherin is associated with pluripotency [37]. These studies 

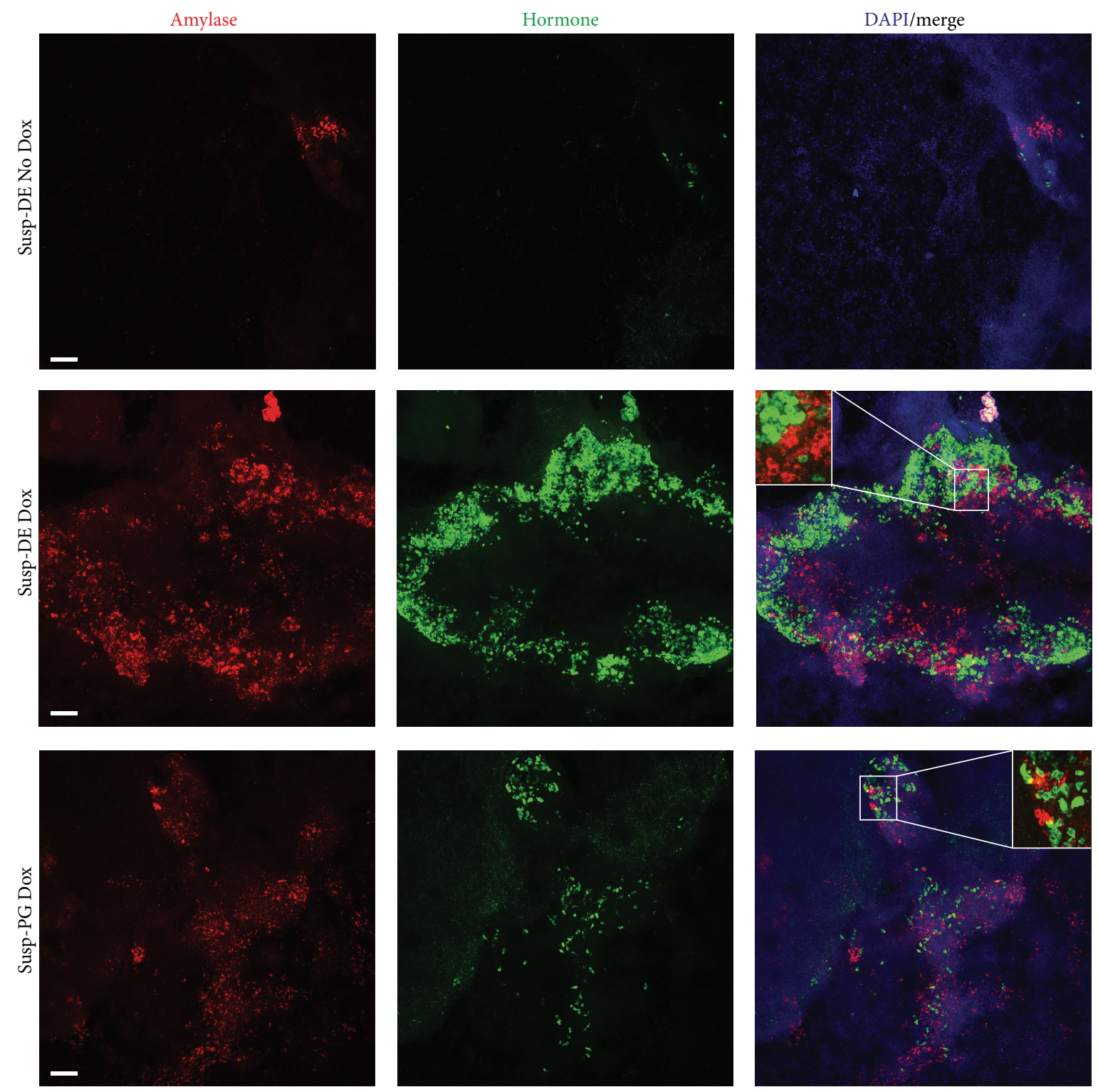

FIgure 7: Cultures that were in suspension during either DE or PG stage when induced with PTFla showed enhanced differentiation to pancreatic cell types. Confocal Z-stacks images at low magnification shown along with DAPI to demonstrate the extent of differentiation between conditions. Susp-DE Dox and Susp-PG Dox cultures had large numbers of Amy ${ }^{+}$and $\mathrm{Horm}^{+}$cells compared to No Dox cultures and attachment cultures (data not shown). Susp-DE Dox cultures had more Amy ${ }^{+}$and $\mathrm{Horm}^{+}$cells than Susp-PG Dox cultures. High magnification insets are shown to indicate cytoplasmic staining of hormones and amylase. Scale bars, $50 \mu \mathrm{m}$.

imply that high cell densities may even inhibit differentiation in general. On the contrary, recent reports show contradictory outcomes; high densities promoted pancreatic differentiation $[38,39]$. Nonetheless, high/low is a relative term: the seeding density in high density cultures $(100,000$ cells $/ \mathrm{cm}^{2}$ ) in the study by Chetty et al. [39] was midway between low and high density cultures in our experiment (Figure 1(a)) and hence suggests a normal relationship (bellcurve) between seeding density versus DE formation.

Induction of PTFla in naïve monolayer DE $\left(\mathrm{SOX}_{1}{ }^{+} \mathrm{FOXA}^{+}\right)$populations resulted in only rare $\mathrm{Pdxl}^{+}$ cell clusters that were more prominent in Nodal-derived than Activin A-derived DE. It has been suggested than Nodal-derived DE is competent for morphogenesis and organ specification, and hence Nodal is a more relevant molecule for ESC differentiation [6]. But these cells never activated the expression of downstream pancreatic genes, indicating that unpatterned endoderm is not a sufficient context for PTFla activity. Hence, we differentiated the monolayer DE further to PF and then induced the expression of PTFla. Importantly, inducing PTFla in the PF population permitted the generation of $\mathrm{PDXl}^{+} \mathrm{NKX}^{+} 1^{+}$pancreatic 


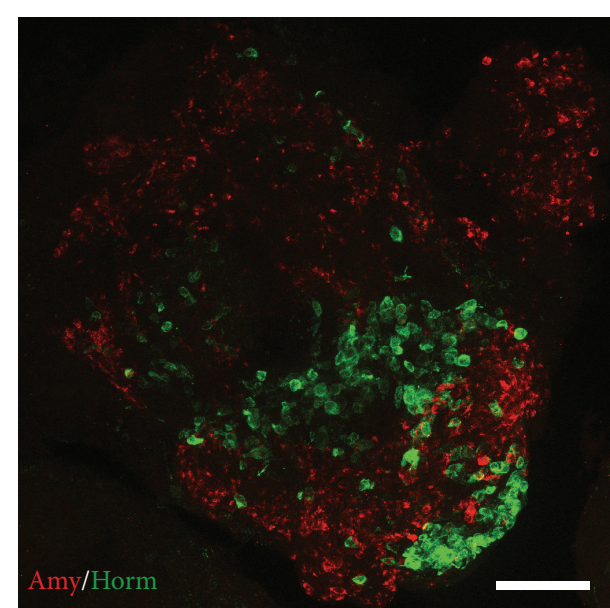

(a)

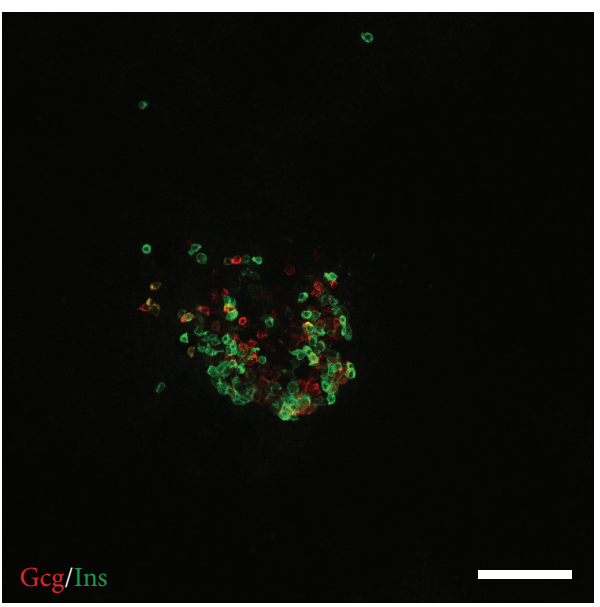

(c)

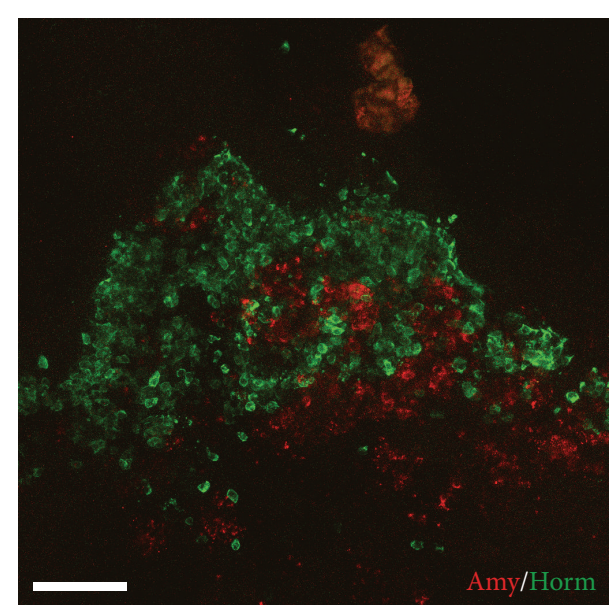

(b)

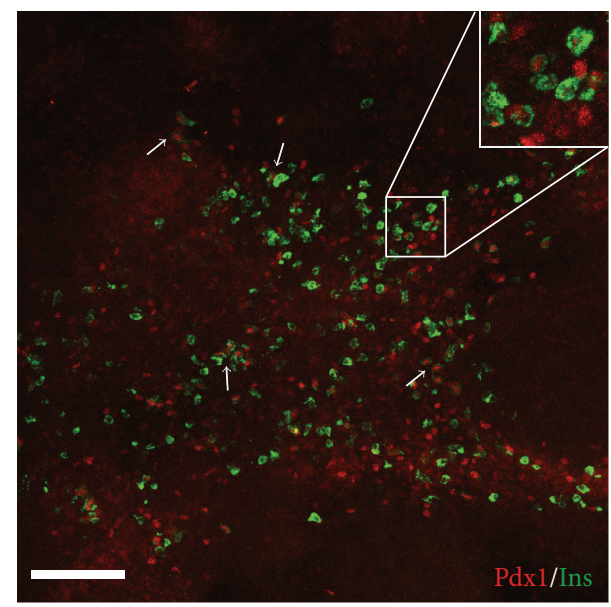

(d)

FIgURE 8: High magnification images of Suspension-DE Dox cultures. $(\mathrm{a}, \mathrm{b}) \mathrm{Horm}^{+}$and $\mathrm{Amy}^{+}$cells are intermingled with each other. In particular amylase and hormone expression are cytoplasmic and do not overlap. (c) Insulin ${ }^{+}$cells are mostly monohormonal but comingled with glucagon $^{+}$cells. (d) Insulin ${ }^{+}$cells express PDX1 in the nucleus. Inset shows higher magnification to indicate nuclear PDX1 in insulin ${ }^{+}$ cells. Arrows point to many insulin ${ }^{+}$cell clusters that have nuclear PDX1. Scale bars, $50 \mu \mathrm{m}$.

progenitors. FGF10 was found to increase the numbers of $\mathrm{PDX1}^{+} \mathrm{NKX} 6.1^{+}$cells synergistically with PTFla activity, and FGF10 alone produced isolated $\mathrm{PDX1}^{+}$cells that did not express NKX6.1. Thus, we have demonstrated the necessity of PTFla expression in the posterior foregut endoderm cells in order to acquire a true pancreatic fate. However, PF-derived $\mathrm{PDX1}^{+} \mathrm{NKX} 61^{+}$cells in these cultures did not undergo terminal differentiation. This could be attributed to several factors, one of them being the presence of FGF10 as FGF10 has been shown to force progenitor arrest maintaining them in a proliferative state and abolishes terminal differentiation $[30,40]$.

While differentiating mESCs in monolayers, we observed some cells spontaneously detaching from the plate and forming floating EB-like populations in suspension especially during the DE and PG stages. Collecting, replating, inducing PTFla, and differentiating these populations led to considerable adult pancreatic cell types in the cultures, including acinar and hormone-expressing cells. Cells that were in suspension during the DE stage most efficiently generated pancreatic cells. In addition, the insulin ${ }^{+}$cells that were produced expressed nuclear PDX1 and did not coexpress glucagon suggesting that they represent mature $\beta$ cells. Growing cells in suspension for a short period of time, somehow, enhanced the potential of PTFla-induced mESCs to progress toward terminal differentiation. 3D aggregates of ESCs or ESC-derived cells possess complex assembly of cellular adhesions, essential for morphogenesis and juxtacrine/paracrine signaling, which are missing in monolayer cultures [32, 33]. Furthermore, additional "inductive" cell type(s) from other germ layers may be generated in $3 \mathrm{D}$ cultures that are essential for proper pancreas development. Moreover, EB-based mESC cultures have been differentiated into DE more successfully than monolayer mESCs [2, 41], with maximum DE markers peaking at Day 4. However, EBs also express $\mathrm{SHH}$, an inhibitor of pancreatic fate, from Day 7 [42]. The findings from these studies could be used to explain our results: cells that are in suspension in both 
Activin A and in low serum differentiate efficiently to DE and are subsequently plated before $\mathrm{SHH}$ induction thereby circumventing pancreatic inhibition. Moreover, the plated EB-derived cells are exposed to SANT-1, a SHH signaling inhibitor, in the PG stage reinforcing the elimination of $\mathrm{SHH}$ activity.

\section{Conclusions}

In essence, we have demonstrated that PTFla activity in an endodermal context, specifically in patterned posterior foregut endoderm, improves the formation of pancreatic progenitor cells in mESCs. Additionally, we found that cell density and ECM substrate affect the output of DE and pancreatic cell types. Above all, PTFla induction in combination with suspension culture formats significantly enhanced differentiation to all adult pancreatic cell types, including insulin $^{+}$cells that were monohormonal. This study asserts the importance of PTFla expression in highly enriched endodermal-derived populations to drive the differentiation of ESCs to $\beta$-like cells efficiently.

$\begin{array}{ll}\text { Abbreviations } \\ \text { 1\% SR: } & \text { 1\% serum replacement } \\ \text { DE: } & \text { Definitive endoderm } \\ \text { ECADH: } & \text { E-cadherin } \\ \text { EB: } & \text { Embryoid body } \\ \text { FOXA2: } & \text { Forkhead box protein A2 } \\ \text { mESC: } & \text { Mouse embryonic stem cells } \\ \text { NKX6.1: } & \text { NK6 homeobox 1 } \\ \text { No-GF: } & \text { No growth factor treated } \\ \text { PDX1: } & \text { Pancreatic and duodenal homeobox 1 } \\ \text { PE: } & \text { Pancreatic endoderm } \\ \text { PF: } & \text { Posterior foregut endoderm } \\ \text { PG: } & \text { Primitive gut } \\ \text { PTF1a: } & \text { Pancreas specific transcription factor la } \\ \text { SOX17: } & \text { Sex determining region Y-boxl7 } \\ \text { Tet-Ptf1a: } & \text { Tetracycline/doxycycline inducible } \\ & \text { mouse embryonic stem cell line. }\end{array}$

\section{Conflict of Interests}

The authors declare that there is no conflict of interests regarding the publication of this paper.

\section{Authors' Contribution}

Gopika G. Nair designed and performed experiments, wrote the paper, and analyzed data. Jon S. Odorico designed the experiments, analyzed data, and wrote and edited the paper.

\section{Acknowledgments}

The authors would like to thank the following individuals for providing access to important reagents: Chris Wright for anti-PDX1 antibody, Ray Macdonald for anti-PTFla antibody, Samuel Pfaff for anti-HLXB9 antibody, and Michael Kyba for
Ainv15 cell line. The authors would also like to acknowledge the CMN Waisman Core facility for training and use of their A1R-Si Nikon Confocal and FACS Calibur machines. Financial support for this project was provided by the following funding: NIH ARRA DK-78889-1A2, JDRF Research Grant no. 2007-75, ADA Innovative Award, Department of Surgery Surgical Associates Award, Vilas Associate Professorship Award, and ROTRF/JDRF Award no. 71612962.

\section{References}

[1] K. A. D’Amour, A. D. Agulnick, S. Eliazer, O. G. Kelly, E. Kroon, and E. E. Baetge, "Efficient differentiation of human embryonic stem cells to definitive endoderm," Nature Biotechnology, vol. 23, no. 12, pp. 1534-1541, 2005.

[2] A. Kubo, K. Shinozaki, J. M. Shannon et al., "Development of definitive endoderm from embryonic stem cells in culture," Development, vol. 131, no. 7, pp. 1651-1662, 2004.

[3] S. Tada, T. Era, C. Furusawa et al., "Characterization of mesendoderm: a diverging point of the definitive endoderm and mesoderm in embryonic stem cell differentiation culture," Development, vol. 132, no. 19, pp. 4363-4374, 2005.

[4] M. Yasunaga, S. Tada, S. Torikai-Nishikawa et al., "Induction and monitoring of definitive and visceral endoderm differentiation of mouse ES cells," Nature Biotechnology, vol. 23, no. 12, pp. 1542-1550, 2005.

[5] M. Hansson, D. R. Olesen, J. M. L. Peterslund et al., "A late requirement for Wnt and FGF signaling during activin-induced formation of foregut endoderm from mouse embryonic stem cells," Developmental Biology, vol. 330, no. 2, pp. 286-304, 2009.

[6] A. E. Chen, M. Borowiak, R. I. Sherwood, A. Kweudjeu, and D. A. Melton, "Functional evaluation of ES cell-derived endodermal populations reveals differences between Nodal and Activin A-guided differentiation," Development, vol. 140, no. 3, pp. 675-686, 2013.

[7] M. Borowiak, R. Maehr, S. Chen et al., "Small molecules efficiently direct endodermal differentiation of mouse and human embryonic stem cells," Cell Stem Cell, vol. 4, no. 4, pp. 348-358, 2009.

[8] H. K. Bone, A. S. Nelson, C. E. Goldring, D. Tosh, and M. J. Welham, "A novel chemically directed route for the generation of definitive endoderm from human embryonic stem cells based on inhibition of GSK-3," Journal of Cell Science, vol. 124, no. 12, pp. 1992-2000, 2011.

[9] A. M. Zorn and M. J. Wells, "Molecular basis of vertebrate endoderm development," in International Review of Cytology, W. J. Kwang, Ed., pp. 49-111, Academic Press, 2007.

[10] D. Dufort, L. Schwartz, K. Harpal, and J. Rossant, "The transcription factor HNF3 $\beta$ is required in visceral endoderm for normal primitive streak morphogenesis," Development, vol. 125, no. 16, pp. 3015-3025, 1998.

[11] M. Kanai-Azuma, Y. Kanai, J. M. Gad et al., "Depletion of definitive gut endoderm in Sox17-null mutant mice," Development, vol. 129, no. 10, pp. 2367-2379, 2002.

[12] M. C. Nostro, F. Sarangi, S. Ogawa et al., "Stage-specific signaling through TGF $\beta$ family members and WNT regulates patterning and pancreatic specification of human pluripotent stem cells," Development, vol. 138, no. 5, pp. 861-871, 2011.

[13] A. Rezania, J. E. Bruin, M. J. Riedel et al., "Maturation of human embryonic stem cell-derived pancreatic progenitors 
into functional islets capable of treating pre-existing diabetes in mice," Diabetes, vol. 61, no. 8, pp. 2016-2029, 2012.

[14] E. Kroon, L. A. Martinson, K. Kadoya et al., "Pancreatic endoderm derived from human embryonic stem cells generates glucose-responsive insulin-secreting cells in vivo," Nature Biotechnology, vol. 26, no. 4, pp. 443-452, 2008.

[15] K. A. D’Amour, A. G. Bang, S. Eliazer et al., "Production of pancreatic hormone-expressing endocrine cells from human embryonic stem cells," Nature Biotechnology, vol. 24, no. 11, pp. 1392-1401, 2006.

[16] Y. Kunisada, N. Tsubooka-Yamazoe, M. Shoji, and M. Hosoya, "Small molecules induce efficient differentiation into insulinproducing cells from human induced pluripotent stem cells," Stem Cell Research, vol. 8, no. 2, pp. 274-284, 2012.

[17] X. Xu, V. L. Browning, and J. S. Odorico, "Activin, BMP and FGF pathways cooperate to promote endoderm and pancreatic lineage cell differentiation from human embryonic stem cells," Mechanisms of Development, vol. 128, no. 7"10, pp. 412-427, 2011.

[18] H. A. Russ, A. V. Parent, J. J. Ringler et al., "Controlled induction of human pancreatic progenitors produces functional beta-like cells in vitro," The EMBO Journal, vol. 34, no. 13, pp. 1759-1772, 2015.

[19] G. Nair and M. Hebrok, "Islet formation in mice and men: lessons for the generation of functional insulin-producing $\beta$ cells from human pluripotent stem cells," Current Opinion in Genetics \& Development, vol. 32, pp. 171-180, 2015.

[20] F. W. Pagliuca, J. R. Millman, M. Gürtler et al., "Generation of functional human pancreatic $\beta$ cells in vitro," Cell, vol. 159, no. 2, pp. 428-439, 2014.

[21] A. Rezania, J. E. Bruin, P. Arora et al., "Reversal of diabetes with insulin-producing cells derived in vitro from human pluripotent stem cells," Nature Biotechnology, vol. 32, no. 11, pp. 1121-1133, 2014.

[22] O. G. Kelly, M. Y. Chan, L. A. Martinson et al., "Cell-surface markers for the isolation of pancreatic cell types derived from human embryonic stem cells," Nature Biotechnology, vol. 29, no. 8, pp. 750-756, 2011.

[23] Y. Kawaguchi, B. Cooper, M. Gannon, M. Ray, R. J. MacDonald, and C. V. E. Wright, "The role of the transcriptional regulator Ptfla in converting intestinal to pancreatic progenitors," Nature Genetics, vol. 32, no. 1, pp. 128-134, 2002.

[24] G. G. Nair, R. K. Vincent, and J. S. Odorico, "Ectopic ptfla expression in murine ESCs potentiates endocrine differentiation and models pancreas development in vitro," Stem Cells, vol. 32, no. 5, pp. 1195-1207, 2014.

[25] J. B. Sneddon, M. Borowiak, and D. A. Melton, "Self-renewal of embryonic-stem-cell-derived progenitors by organ-matched mesenchyme," Nature, vol. 491, no. 7426, pp. 765-768, 2012.

[26] R. Vincent, N. Treff, M. Budde, Z. Kastenberg, and J. Odorico, "Generation and characterization of novel tetracyclineinducible pancreatic transcription factor-expressing murine embryonic stem cell lines," Stem Cells and Development, vol. 15, no. 6, pp. 953-962, 2006.

[27] B. W. Kahan, L. M. Jacobson, D. A. Hullett et al., "Pancreatic precursors and differentiated islet cell types from murine embryonic stem cells," Diabetes, vol. 52, no. 8, pp. 2016-2024, 2003.

[28] A. S. Bernardo, C. H.-H. Cho, S. Mason et al., "Biphasic induction of Pdxl in mouse and human embryonic stem cells can mimic development of pancreatic $\beta$-cells," STEM CELLS, vol. 27, no. 2, pp. 341-351, 2009.
[29] A. Hart, S. Papadopoulou, and H. Edlund, "Fgf10 maintains notch activation, stimulates proliferation, and blocks differentiation of pancreatic epithelial cells," Developmental Dynamics, vol. 228, no. 2, pp. 185-193, 2003.

[30] G. A. Norgaard, J. N. Jensen, and J. Jensen, "FGF10 signaling maintains the pancreatic progenitor cell state revealing a novel role of Notch in organ development," Developmental Biology, vol. 264, no. 2, pp. 323-338, 2003.

[31] P. Jacquemin, H. Yoshitomi, Y. Kashima, G. G. Rousseau, F. P. Lemaigre, and K. S. Zaret, "An endothelial-mesenchymal relay pathway regulates early phases of pancreas development," Developmental Biology, vol. 290, no. 1, pp. 189-199, 2006.

[32] A. M. Bratt-Leal, R. L. Carpenedo, and T. C. McDevitt, "Engineering the embryoid body microenvironment to direct embryonic stem cell differentiation," Biotechnology Progress, vol. 25, no. 1, pp. 43-51, 2009.

[33] D. ten Berge, W. Koole, C. Fuerer, M. Fish, E. Eroglu, and R. Nusse, "Wnt signaling mediates self-organization and axis formation in embryoid bodies," Cell Stem Cell, vol. 3, no. 5, pp. 508-518, 2008.

[34] Y. Xu, X. Zhu, H. S. Hahm et al., "Revealing a core signaling regulatory mechanism for pluripotent stem cell survival and self-renewal by small molecules," Proceedings of the National Academy of Sciences of the United States of America, vol. 107, no. 18, pp. 8129-8134, 2010.

[35] J. Cai, C. Yu, Y. Liu et al., "Generation of homogeneous PDX1+ pancreatic progenitors from human ES cell-derived endoderm cells," Journal of Molecular Cell Biology, vol. 2, no. 1, pp. 50-60, 2010.

[36] J. J. Otero, W. Fu, L. Kan, A. E. Cuadra, and J. A. Kessler, " $\beta$-Catenin signaling is required for neural differentiation of embryonic stem cells," Development, vol. 131, no. 15, pp. 35453557, 2004.

[37] A. M. Arias, F. Faunes, P. Hayward et al., "A membraneassociated $\beta$-catenin/Oct 4 complex correlates with groundstate pluripotency in mouse embryonic stem cells," Development, vol. 140, no. 6, pp. 1171-1183, 2013.

[38] B. K. Gage, T. D. Webber, and T. J. Kieffer, "Initial cell seeding density influences pancreatic endocrine development during in vitro differentiation of human embryonic stem cells," PLoS ONE, vol. 8, no. 12, Article ID e82076, 2013.

[39] S. Chetty, F. W. Pagliuca, C. Honore, A. Kweudjeu, A. Rezania, and D. A. Melton, "A simple tool to improve pluripotent stem cell differentiation," Nature Methods, vol. 10, no. 6, pp. 553-556, 2013.

[40] S. Kobberup, M. Schmerr, M.-L. Dang et al., "Conditional control of the differentiation competence of pancreatic endocrine and ductal cells by Fgf10," Mechanisms of Development, vol. 127, no. 3-4, pp. 220-234, 2010.

[41] F. Li, Z. He, Y. Li et al., "Combined Activin A/LiCl/Noggin treatment improves production of mouse embryonic stem cellderived definitive endoderm cells," Journal of Cellular Biochemistry, vol. 112, no. 4, pp. 1022-1034, 2011.

[42] J. K. Mfopou, V. De Groote, X. Xu, H. Heimberg, and L. Bouwens, "Sonic Hedgehog and other soluble factors from differentiating embryoid bodies inhibit pancreas development," Stem Cells, vol. 25, no. 5, pp. 1156-1165, 2007. 

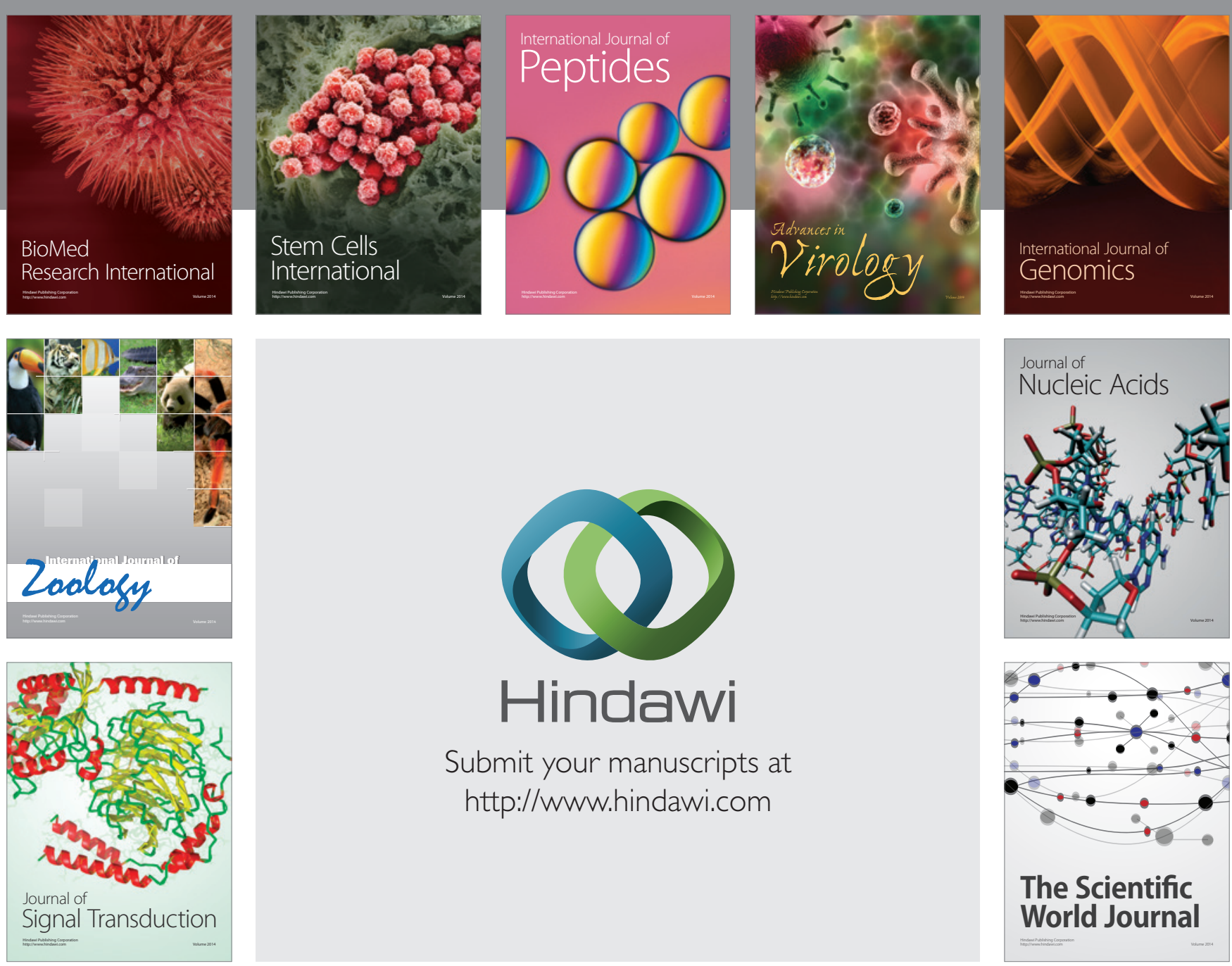

Submit your manuscripts at

http://www.hindawi.com
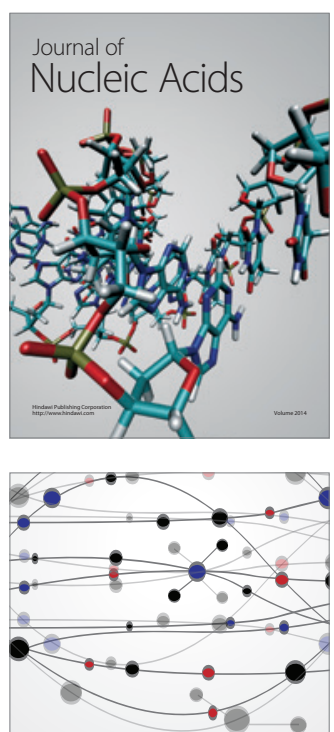

The Scientific World Journal
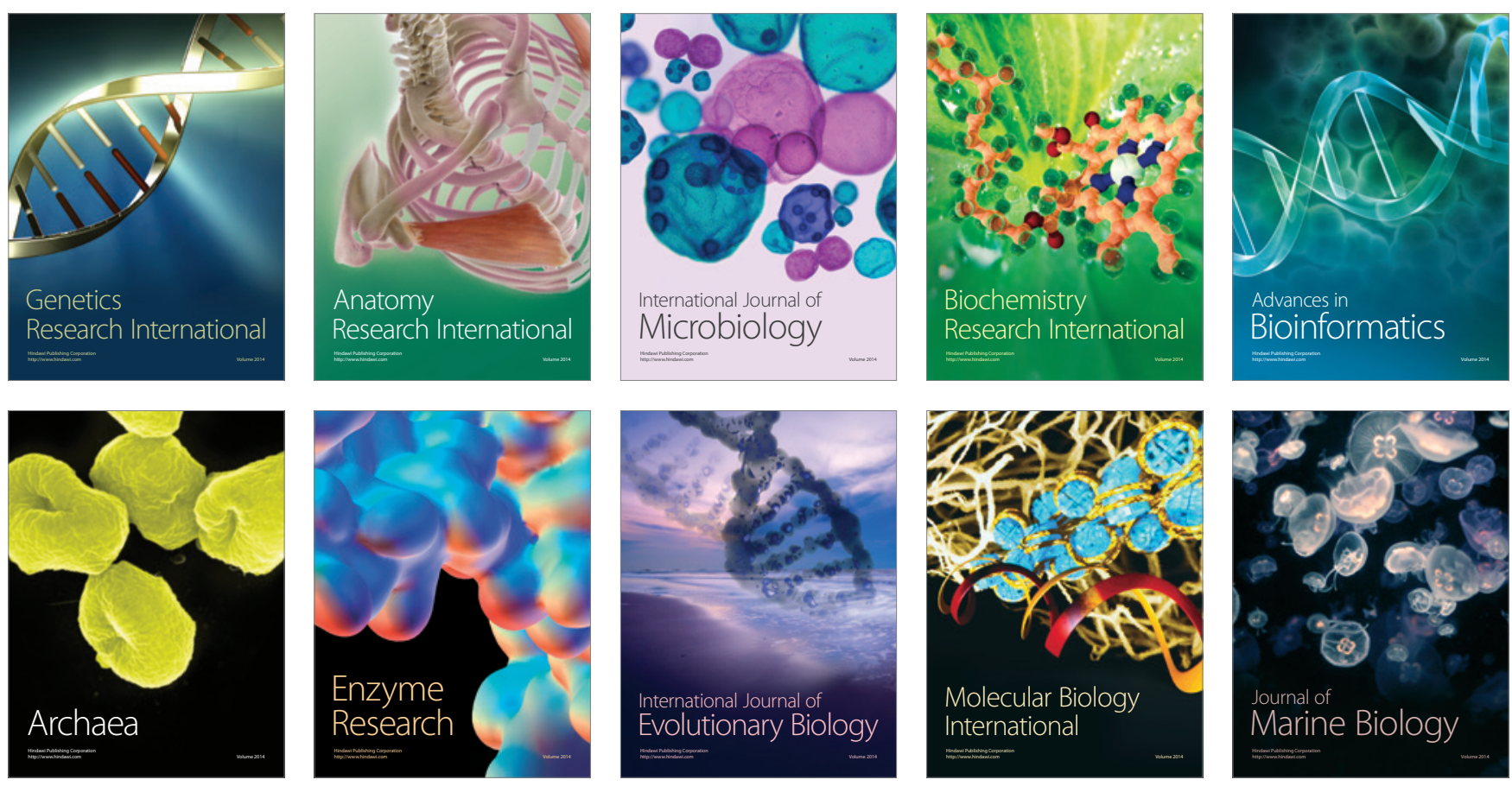